\title{
Equivariant plateau problems
}

\author{
Graham Smith
}

Received: 23 October 2006 / Accepted: 24 October 2008 / Published online: 28 November 2008

(C) The Author(s) 2008. This article is published with open access at Springerlink.com

\begin{abstract}
Let $(M, Q)$ be a compact, three dimensional manifold of strictly negative sectional curvature. Let $(\Sigma, P)$ be a compact, orientable surface of hyperbolic type (i.e. of genus at least two). Let $\theta: \pi_{1}(\Sigma, P) \rightarrow \pi_{1}(M, Q)$ be a homomorphism. Generalising a recent result of Gallo, Kapovich and Marden concerning necessary and sufficient conditions for the existence of complex projective structures with specified holonomy to manifolds of non-constant negative curvature, we obtain necessary conditions on $\theta$ for the existence of a so called $\theta$-equivariant Plateau problem over $\Sigma$, which is equivalent to the existence of a strictly convex immersion $i: \Sigma \rightarrow M$ which realises $\theta$ (i.e. such that $\theta=i_{*}$ ).
\end{abstract}

Keywords Kleinian groups - Fuchsian groups - Plateau problem - Complex projective structures · Immersions

Mathematics Subject Classification (2000) $57 \mathrm{M} 50 \cdot 30 \mathrm{~F} 10 \cdot 30 \mathrm{~F} 40 \cdot 32 \mathrm{G} 15$

\section{Introduction}

In this paper, we study means of obtaining constant curvature realisations of homomorphisms of fundamental groups of surfaces into fundamental groups of compact, three dimensional manifolds of strictly negative sectional curvature.

Let $(M, Q)$ be a pointed, compact three dimensional Riemannian manifold of strictly negative sectional curvature. Let $(\Sigma, P)$ be a closed (orientable) surface of hyperbolic type (i.e. of genus at least 2). Let $(\tilde{M}, \tilde{Q})$ be the universal cover of $(M, Q)$. Let $\theta: \pi_{1}(\Sigma, P) \rightarrow$ $\pi_{1}(M, Q)$ be a representation of $\pi_{1}(\Sigma, P)$ in $\pi_{1}(M, Q)$. We observe that it is in many ways preferable to work in the framework of pointed manifolds. Firstly, $\pi_{1}(M, Q)$ is canonically defined in terms of $(M, Q)$, and, secondly, the action of $\pi_{1}(M, Q)$ over $(\tilde{M}, \tilde{Q})$ is well defined. 
$\tilde{M}$ is a Hadamard manifold. We denote by $\partial_{\infty} \tilde{M}$ its ideal boundary, and we make the following definition:

Definition 1.0.1 Let $(\tilde{\Sigma}, \tilde{P})$ be the universal cover of $(\Sigma, P)$. Let $\theta: \pi_{1}(\Sigma, P) \rightarrow \pi_{1}(M, Q)$ be a homomorphism. A $\theta$-equivariant Plateau problem over $\Sigma$ is a function $\varphi: \tilde{\Sigma} \rightarrow \partial_{\infty} \tilde{M}$ such that, for all $\gamma \in \pi_{1}(\Sigma, P)$ :

$$
\varphi \circ \gamma=\theta(\gamma) \circ \varphi
$$

Such structures are introduced and discussed in detail by Labourie in [5] and by the author in [8] and [7].

The group $\pi_{1}(M, Q)$ acts canonically on $\tilde{M} \cup \partial_{\infty} \tilde{M}$. We emphasize that $\pi_{1}(M, Q)$ acts on $\tilde{M} \cup \partial_{\infty} \tilde{M}$ from the right. Thus, throughout this entire paper, composition is to be read from left to right. A subgroup $\Gamma$ of $\pi_{1}(M, Q)$ is said to be non-elementary if and only if it has no fixed points in $\tilde{M} \cup \partial_{\infty} \tilde{M}$, and we will say that it is elementary otherwise. In our case, the only elementary subgroups of $\pi_{1}(M, Q)$ are the trivial group, and isomorphic copies of $\mathbb{Z}$.

Let $\mathrm{Homeo}_{0}\left(\partial_{\infty} \tilde{M}\right)$ denote the connected component of the group of homeomorphisms of $\partial_{\infty} \tilde{M}$ which contains the identity. The group $\pi_{1}(M, Q)$ acts faithfully over $\partial_{\infty} \tilde{M}$, and may thus be considered as a subgroup of $\operatorname{Homeo}_{0}\left(\partial_{\infty} \tilde{M}\right)$. Let $\widetilde{H}^{\circ \mathrm{meo}_{0}}\left(\partial_{\infty} \tilde{M}\right)$ be the universal cover of $\operatorname{Homeo}_{0}\left(\partial_{\infty} \tilde{M}\right)$, and let $\pi: \operatorname{Homeo}_{0}\left(\partial_{\infty} \tilde{M}\right) \rightarrow \operatorname{Homeo}_{0}\left(\partial_{\infty} \tilde{M}\right)$ be the canonical projection. We have the following short exact sequence:

$$
0 \rightarrow \mathbb{Z}_{2} \stackrel{i}{\rightarrow} \mathrm{Homeo}_{0}\left(\partial_{\infty} \tilde{M}\right) \stackrel{\pi}{\rightarrow} \operatorname{Homeo}_{0}\left(\partial_{\infty} \tilde{M}\right) \rightarrow 0
$$

For $\Gamma$ an arbitrary group, and for $\varphi: \Gamma \rightarrow \operatorname{Homeo}_{0}\left(\partial_{\infty} \tilde{M}\right)$ a homomorphism, we define a lifting of $\varphi$ in $\mathrm{Homeo}_{0}\left(\partial_{\infty} \tilde{M}\right)$ to be a homomorphism $\hat{\varphi}: \Gamma \rightarrow \mathrm{HomeO}_{0}\left(\partial_{\infty} \tilde{M}\right)$ such that $\pi \circ \hat{\varphi}=\varphi$. We say that $\varphi$ lifts if such a lifting may be found.

Returning to the special case where $M$ is of constant sectional curvature equal to -1 , we recall an existence result, obtained by Gallo, Kapovich and Marden [4], which, when translated into our framework, may be expressed as follows:

Theorem 1.0.2 (Gallo, Kapovich and Marden [4]) Suppose that $M$ is of constant sectional curvature equal to -1 . Then, there exists a $\theta$-equivariant Plateau problem $\varphi$ over $\Sigma$ if and only if $\theta$ is non-elementary and lifts.

The requirement that $\theta$ be non-elementary arises from the fact that $\varphi$ defines a $\operatorname{PSL}(2, \mathbb{C})$ structure over the surface $\Sigma$. If $\theta$ were elementary, then it would be a subset either of the rotation group or of the affine group. In the former case, it would define an $\mathrm{SO}$ (3) structure over $\Sigma$, which would induce a metric of constant positive curvature over $\Sigma$, which is excluded by the Gauss-Bonnet theorem. Likewise, in the latter case, it would define an affine structure over $\Sigma \backslash X$, where $X=\pi\left(\varphi^{-1}(\{\infty\})\right)$, and $\infty$ is assumed to be the unique point in the sphere preserved by the image of $\theta$. By compactness of $\Sigma, X$ is finite, and since each critical point lies in a single affine chart, the Gauss-Bonnet theorem may again be used to obtain a contradiction.

This theorem yields the following interesting corollary:

Corollary 1.0.3 Suppose that $M$ has constant sectional curvature equal to -1 . There exists a locally convex immersion $i: \Sigma \rightarrow M$ such that $\theta=i_{*}$ if and only if $\theta$ is non-elementary and lifts. 
The objective of this paper is to obtain an analogue of this result in the more general case where $M$ is only of strictly negative sectional curvature. The main result of this paper is the following:

Theorem 1.0.4 Suppose that $(M, Q)$ is a pointed, compact manifold of strictly negative sectional curvature. Let $(\Sigma, P)$ be a pointed, compact surface of hyperbolic type (i.e. of genus at least two). Let $\theta: \pi_{1}(\Sigma, P) \rightarrow \pi_{1}(M, Q)$ be a homomorphism. Suppose that $\theta$ is nonelementary and may be lifted to a homomorphism $\hat{\theta}$ of $\pi_{1}(\Sigma, P)$ into the group $\mathrm{HomeO}_{0}$ $\left(\partial_{\infty} \tilde{M}\right)$. Then there exists an equivariant Plateau problem for $\theta$.

This result permits us to obtain a locally convex realisation of $\theta$ :

Theorem 1.0.5 If $\theta$ is non-elementary and lifts, then there exists a convex immersion $i$ : $\Sigma \rightarrow M$ such that $\theta=i_{*}$.

Theorem 1.0.4 is, in certain respects, a generalisation to non-constant curvature of Theorem 1.0.2, and will be proven using essentially the same techniques, following the observation that many of the algebraic results used in [4], which thus depend on the structure of $\mathbb{P} S L(2, \mathbb{C})=\operatorname{Isom}\left(\mathbb{H}^{3}\right)$, may be expressed in a purely topological manner, and may thus be applied to $\operatorname{Isom}(\tilde{M})$.

Our approach differs from that of Gallo, Kapovich and Marden in various respects. By far the most significant, however, is our treatment of the algebraic obstruction. In [4], the complex structure allows the algebraic obstruction to be rapidly treated using classical techniques associated to index theory. Since the case that we study is purely topological, such powerful tools are no longer available, and a much deeper understanding of the algebraic properties of the construction described in [4] is required. This forms the content of Sect. 4 and Appen$\operatorname{dix} \mathrm{A}$. The more perspicacious reader will also observe that this deeper understanding allows us to simplify the pants decomposition of [4], in that it is no longer necessary that it take the form of a tree.

The remaining differences between our approaches involve the sort of simplifications that one would expect to arise from any in-depth study of such a significant work. First, in Sect. 3, we adopt the perspective that the homomorphism, $\theta$, as opposed to the paths that we study, is changed by the Dehn twists. These approaches are trivially logically equivalent, but we believe that our perspective facilitates the reading of this paper by hiding the complexity of the construction within the algebra. Moreover, we construct the Dehn twists used in [4] as compositions of much simpler Dehn twists. We believe that this also facilitates the understanding of our proofs.

Finally, in Sect. 2, since we only work with mappings of hyperbolic type, we may bypass most of the technically complicated algebraic reasoning employed in [4] to obtain the algebraic results necessary for the construction of Schottky groups. The simpler conditions of the case we study allow us to prove these results in terms of elementary arguments concerning the fixed point sets of hyperbolic mappings and a general property of Hadamard manifolds with sectional curvature bounded above by $k<0$. This alternative approach is crucial for our results, since the algebraic approach used in [4] is only valid for the Möbius group. In a way, this part of our work may also be considered as an illustrative example, facilitating the understanding of Gallo, Kapovich and Marden's result without requiring an in-depth understanding of the technical complexity of their own paper.

This paper may be divided into three parts. In the first part, comprising Sects. 2 and 3, we show how to obtain a pants decomposition of a surface where the image of the fundamental group of each pant under $\theta$ is a Schottky group. This part more or less exactly follows 
the corresponding sections of [4], adapted and simplified within our current context. The argument is very technical, but elementary. It involves the repeated application of a number of simple geometric manipulations, much like solving a Rubik's cube. Thus, in Sect. 2, we obtain algebraic results concerning the construction of Schottky groups. In Sect. 3, we use Dehn twists to construct the desired pants decomposition of $\Sigma$. Here we begin to develop the algebraic perspective which will be used in the later sections. In particular, we define the auxiliary notions of bindings and of marked surfaces with holonomy in a given group. These are essentially book keeping measures allowing us to record the information which is lost during the decomposition process. They are subsequently used in the construction in Sect. 5.

In the second part, comprising Sect. 4, we study the geometry of the actions of Schottky groups on the sphere. We define invariant domains of Schottky groups as subsets of the sphere, and exhibit how these domains define a number of algebraic structures associated to the Schottky groups. We thus describe diverse algebraic objects, including universal covers of homeomorphism groups, braid groups and the homologies of tori, all of which are combined to describe the algebraic obstruction (Lemma 4.1.1) to producing suitable solutions over each pant which may subsequently be glued together to prove Theorem 1.0.4. This is the most original, and perhaps the hardest, part of the paper.

In the third part, comprising Sect. 5, we return to the glueing construction of [4], showing how the algebraic tools developed in Sect. 4 yield a more precise understanding of the algebraic behaviour of the glueing operations. Thus, we use invariant domains to construct $\theta$-equivariant Plateau problems over pants subject to certain algebraic conditions, and we then glue these functions together to obtain a proof of Theorems 1.0.4 and 1.0.5.

Finally, in the Appendix, we prove the homeomorphism equivalence of Schottky groups. In particular, this permits us to construct invariant domains for any Schottky group.

The main ideas of this paper were already outlined in the fourth chapter of the author's doctoral thesis [7]. This paper presents a significant clarification of these results.

I would like to thank François Labourie for having drawn my attention to this problem, Kevin Costello for help with algebraic topology, and Frédéric Leroux, for having introduced me to the results of Friberg. I would also like to thank the Max Planck Institute for Mathematics in the Sciences in Leipzig for providing the comfort required to write the English version of this paper.

\section{Schottky groups}

\subsection{Introduction}

Throughout this section, $M$ will denote a three dimensional Hadamard manifold. The construction that we will carry out in the sequel makes considerable use of Schottky groups. We define such groups as follows:

Definition 2.1.1 Let $C_{a}^{-}, C_{a}^{+}, C_{b}^{-}$and $C_{b}^{+}$be disjoint Jordan curves in the sphere $S^{2}$ oriented such that each one of these curves is situated in the exterior of the three others. We denote the group of homeomorphisms of $S^{2}$ which preserve orientation by $\operatorname{Homeo}_{0}\left(S^{2}\right)$. We will adopt the convention that this group acts on $S^{2}$ from the right. A Schottky group is a subgroup $\Gamma$ of $\operatorname{Homeo}_{0}\left(S^{2}\right)$ generated by two elements $a$ and $b$ such that:

$$
\operatorname{Ext}\left(C_{a}^{-}\right) \cdot a=\operatorname{Int}\left(C_{a}^{+}\right), \quad \operatorname{Ext}\left(C_{b}^{-}\right) \cdot b=\operatorname{Int}\left(C_{b}^{+}\right) .
$$


We will refer to the curves $\left(C_{a}^{ \pm}, C_{b}^{ \pm}\right)$as generating circles for the Schottky group $\Gamma$ with respect to the pair of generators $(a, b)$.

Let $\alpha: M \rightarrow M$ be an isometry of $M$. The mapping $\alpha$ may be extended to a homeomorphism of $M \cup \partial_{\infty} M$. A mapping is hyperbolic if and only if it has precisely two fixed points in $\partial_{\infty} M$ and no fixed points in $M$ (see [2]). In the sequel, we will work with actions of fundamental groups of compact manifolds of strictly negative sectional curvature on the universal covers of these manifolds. In such subgroups $\Gamma \subseteq \operatorname{Isom}(M)$, every element that is different to the identity is necessarily hyperbolic.

In this section, we show how Schottky subgroups of $\operatorname{Homeo}_{0}\left(\partial_{\infty} M\right)$ may be constructed. The following results are of a technical nature, and their geometric significance will become clearer when they are applied in Sect. 3.

Proposition 2.1.2 Let $M$ be a Hadamard manifold. Let $\Gamma$ be a subgroup of the group of isometries of $M$ consisting only of hyperbolic elements (and the identity). Let $\alpha, \beta, \xi$ and $\eta$ be elements of $\Gamma$ such that the subgroup $\langle\alpha, \beta\rangle$ of $\Gamma$ is non-elementary. There exists $K \in \mathbb{N}$ and a function $N:\{|n| \geqslant K\} \rightarrow \mathbb{N}$ such that, for all $|k| \geqslant K$ and for all $|n| \geqslant N(k)$ :

(1) $\beta \alpha^{k}$ is hyperbolic,

(2) $\delta_{k}=\eta \beta \alpha^{k}$ is hyperbolic,

(3) $\delta_{k}^{n} \alpha$ is hyperbolic and has no fixed point in common with $\beta \alpha^{k}$, and

(4) $\delta_{k}^{n} \xi$ is hyperbolic.

In other words, $\beta \alpha^{k}, \delta_{k}, \delta_{k}^{n} \alpha$ and $\delta_{k}^{n} \xi$ are all different to the identity.

Remark We observe that this result (along with the other results in this subsection) makes no use of the dimension of the Hadamard manifold, except in the definition of the Schottky groups. In higher dimensions, Schottky groups may be defined using topological spheres instead of circles, and the same results therefore hold.

Proposition 2.1.3 Let $M$ be a Hadamard manifold. Let $\Gamma$ be a subgroup of the group of isometries of $M$ consisting only of hyperbolic elements (and the identity). Let $\alpha, \beta, \gamma$ and $\eta$ be elements of $\Gamma$ such that the subgroup $\langle\alpha, \beta\rangle$ generated by $\alpha$ and $\beta$ is non-elementary. Suppose moreover that $\xi$ and $\eta$ are both hyperbolic.

There exists $K \in \mathbb{N}$ and a function $N:\{|n| \geqslant K\} \rightarrow \mathbb{N}$ such that, either:

(i) for all $|k| \geqslant K$ and for all $|n| \geqslant N(k)$, if $\delta_{k}=\eta \beta \alpha^{k}$, then $\left\langle\delta_{k}^{n} \xi \delta_{k}^{-n}, \eta\right\rangle$ is a Schottky group, or

(ii) for all $|k| \geqslant K$ and for all $|n| \geqslant N(k)$, if $\delta_{k}=\xi \beta \alpha^{k}$, then $\left\langle\delta_{k}^{n} \eta \delta_{k}^{-n}, \xi\right\rangle$ is a Schottky group.

Proposition 2.1.4 Let $M$ be a Hadamard manifold. Let $\Gamma$ be a subgroup of the group of isometries of $M$ consisting only of hyperbolic elements (and the identity). Let $\alpha, \beta$ be elements of $\Gamma$ such that the subgroup $\langle\alpha, \beta\rangle$ is non-elementary.

For all $\sigma \in \Gamma$, there exists $K \in \mathbb{N}$ such that, for all $|k| \geqslant K, J_{k}=\sigma^{k} \alpha \sigma^{-k} \beta$ is hyperbolic.

Proposition 2.1.5 Let $M$ be a Hadamard manifold. Let $\Gamma$ be a subgroup of the group of isometries of $M$ consisting only of hyperbolic elements (and the identity). Let $\alpha, \beta, \xi$ and $\eta$ be elements of $\Gamma$ such that:

(i) the subgroup $\langle\alpha, \beta\rangle$ is non-elementary,

(ii) $\alpha \beta \alpha^{-1} \beta^{-1}=\eta \xi$, 
(iii) the subgroup $\langle\xi, \eta\rangle$ is a Schottky group,

(iv) $\xi \beta$ is not equal to the identity.

Then there exists $k$ and $n$ such that, if we define $\gamma_{k}$ and $\delta_{k}$ by:

$$
\gamma_{k}=\beta \alpha^{k}, \quad \delta_{k}=\xi \gamma_{k},
$$

then, $\left\langle\gamma_{k},\left(\delta_{k}^{n} \alpha\right)^{-1} \gamma_{k}^{-1}\left(\delta_{k}^{n} \alpha\right)\right\rangle$ and $\left\langle\xi, \delta_{k}^{-n} \eta \delta_{k}^{n}\right\rangle$ are Schottky groups:

In the second part of this section, we review properties of isometries of Hadamard manifolds. In the third part, we study properties of conical open sets about geodesics. In the fourth part, we prove general results about the construction of Schottky groups, and in the final part, we prove the above propositions.

\subsection{Hyperbolic isometries of Hadamard manifolds}

It is well known that a Hadamard manifold is canonically diffeomorphic (under the exponential mapping) to its tangent space over any given point. Similarly, its ideal boundary is canonically homeomorphic to the unit sphere in the tangent space over any given point. We have the following analogous result for normal bundles over geodesics:

Lemma 2.2.1 Let $M$ be a Hadamard manifold of sectional curvature bounded above by $-\epsilon<0$. Let $\gamma$ be a geodesic in M. Let $N_{\gamma}$ be the normal bundle over $\gamma$. In other words:

$$
N_{\gamma}=\left\{X \in T_{\gamma(t)} M \mid t \in \mathbb{R}, X_{p} \perp \partial_{t} \gamma(t)\right\} .
$$

The restriction of the exponential mapping to $N_{\gamma}$ is a diffeomorphism between $N_{\gamma}$ and $M$. Likewise, let $\Sigma_{\gamma}$ be the normal sphere bundle over $\gamma$ contained in $N_{\gamma}$. In other words:

$$
\Sigma_{\gamma}=\left\{X \in N_{\gamma} \mid\|X\|=1\right\} .
$$

Let us define $\partial_{\infty} \gamma$ by:

$$
\partial_{\infty} \gamma=\{\gamma(-\infty), \gamma(\infty)\} .
$$

The restriction of the Gauss-Minkowski mapping defines a homeomorphism between $\Sigma_{\gamma}$ and $\partial_{\infty} M \backslash \partial_{\infty} \gamma$.

Remark We recall that if $U M$ if the unitary bundle of $M$, then the Gauss-Minkowski mapping $\vec{n}: U M \rightarrow \partial_{\infty} M$ is defined such that:

$$
\vec{n}\left(\partial_{t} \gamma(0)\right)=\gamma(+\infty)
$$

for any unit speed geodesic $\gamma: \mathbb{R} \rightarrow M$.

Let us suppose that the sectional curvature of $M$ is bounded above by $-\epsilon<0$. Let $p_{ \pm}$be the fixed points of $\alpha$. There exists a unique oriented geodesic $\gamma$ such that $\gamma(-\infty)=p_{-}$and $\gamma(+\infty)=p_{+}$. This geodesic is preserved by $\alpha$. Moreover, there exists $T_{0} \in \mathbb{R}$ such that, for all $t \in \mathbb{R}$ :

$$
(\alpha \circ \gamma)(t)=\gamma\left(t+T_{0}\right)
$$

We define $\|\alpha\|$, the minimal displacement of $\alpha$, by $\|\alpha\|=\left|T_{0}\right|$. We may show that:

$$
\|\alpha\|=\operatorname{Inf}_{x \in M} d(x, x \cdot \alpha) .
$$


2.3 Conical open sets about geodesics

Let $p$ be a point in $\partial_{\infty} M$. For $q \in M \cup \partial_{\infty} M$, and $r \in M$, we denote by $\widehat{p r q}$ the angle at $r$ between the geodesics joining $r$ to $p$ and $r$ to $q$ respectively. For $\theta \in(0, \pi)$, we consider the neighbourhood $\Omega_{\theta}(p ; r)$ of $p$ in $M \cup \partial_{\infty} M$ given by:

$$
\Omega_{\theta}(p ; r)=\left\{q \in M \cup \partial_{\infty} M \text { s.t. } \widehat{p r q}<\theta\right\} .
$$

We will require the following technical but elementary result:

Lemma 2.3.1 For all $k>0$ there exists a continuous function $\Delta_{k}: \mathbb{R}^{+} \times(0, \pi) \rightarrow(0, \pi)$ with the following properties. If $M$ is a Hadamard manifold with curvature less than or equal to $-k$, and if $\gamma: \mathbb{R} \rightarrow M$ is a geodesic in $M$, then, for all $R \in \mathbb{R}^{+}$and for all $t \in \mathbb{R}$ :

$$
\Omega_{\theta}(\gamma(-\infty), \gamma(t)) \subseteq \Omega_{\Delta_{k}(R, \theta)}(\gamma(-\infty), \gamma(t+R)) .
$$

\section{Moreover:}

(i) $\Delta_{k}(R, \theta)$ is strictly increasing in $\theta$ and strictly decreasing in $R$,

(ii) $\Delta_{k}(R, \theta)$ tends to zero (locally uniformly in $\theta$ ) as $R$ tends to infinity, and

(iii) for all $R$ and for all $\theta, \Delta_{k}(R, \theta)<\theta$.

Proof Let $M_{k}$ be the two dimensional Hadamard manifold of constant curvature equal to $-k$. Let $X Y Z$ be a triangle in $M_{k} \cup \partial_{\infty} M_{k}$ such that:

$$
X \in \partial_{\infty} M_{k}, \quad Y Z=R, \quad \widehat{X Y Z}=\pi-\theta .
$$

We define $\Delta_{k}(R, \theta)$ by:

$$
\Delta_{k}(R, \theta)=\widehat{Y Z X} \text {. }
$$

Let $q$ be a point in $\Omega_{\theta}(\gamma(-\infty) ; \gamma(t))$. Let us denote $A=\gamma(t), B=\gamma(t+R)$ and $C=q$. Let $A^{\prime} B^{\prime} C^{\prime}$ be the comparison triangle in $M_{k}$ such that:

$$
A^{\prime} B^{\prime}=A B, \quad A^{\prime} C^{\prime}=A C, \quad \widehat{C^{\prime} A^{\prime} B^{\prime}}=\widehat{C A B} .
$$

Let $C^{\prime \prime}$ be the point in $\partial_{\infty} M$ obtained by following the geodesic running from $A^{\prime}$ to $C^{\prime}$ to its end point in $\partial_{\infty} M_{k}$. We have:

$$
\widehat{C B A} \leqslant \widehat{C^{\prime} B^{\prime} A^{\prime}} \leqslant \widehat{C^{\prime \prime} B^{\prime} A^{\prime}} \leqslant \widehat{Y Z X}=\Delta_{k}(R, \theta) .
$$

The result now follows.

We also require the following result.

Lemma 2.3.2 Let $p$ be a point in $\partial_{\infty} M$. Let $\Omega$ be a neighbourhood of $p$ in $M \cup \partial_{\infty} M$. There exists a neighbourhood $U$ of $p$ in $\partial_{\infty} \Omega$ such that, for $\gamma$ in $M$ a geodesic:

$$
\gamma(-\infty), \gamma(+\infty) \in U \Rightarrow \gamma \subseteq \Omega \text {. }
$$

Proof Indeed, otherwise, by constructing a sequence and taking limits, we obtain a geodesic $\gamma \in M$ such that:

$$
\gamma(0) \in \partial \Omega \cap M, \quad \gamma(-\infty), \gamma(+\infty)=p
$$

This is absurd. 


\subsection{Constructing Schottky groups}

We now obtain the following result which shows how Schottky groups may be constructed in general:

Lemma 2.4.1 Let $k>0$ be a real number and let $M$ be a Hadamard manifold with curvature less than $-k$. Let $\alpha$ be a hyperbolic isometry of $M$ having $p_{+}$and $p_{-}$as attractive and repulsive fixed points respectively. Let $p_{0}$ be a point distinct from $p_{ \pm}$. For every $B>0$, there exists a neighbourhood $\Omega$ of $p_{0}$ in $\partial_{\infty} M$ such that if $q_{ \pm} \in \Omega$ and if $\beta$ is a hyperbolic isometry of $M$ having $q_{ \pm}$as fixed points such that $\|\beta\|>B$, then the subgroup $\langle\alpha, \beta\rangle$ of $\operatorname{Isom}(M)$ generated by $\alpha$ and $\beta$ is a Schottky group.

Proof Let us also denote by $\alpha$ the unique geodesic in $M$ such that $\alpha( \pm \infty)=p_{ \pm}$. Let $\Sigma_{\alpha}$ be the normal sphere bundle over $\alpha$. Let $\vec{n}: \Sigma_{\alpha} \rightarrow \partial_{\infty} M$ be the Gauss-Minkowski mapping. By Lemma 2.2.1, there exists a unique vector $X \in \Sigma_{\alpha}$ such that $\vec{n}(X)=p_{0}$. We suppose that $\alpha$ is normalised such that $X$ lies in the fibre over $\alpha(0)$. We define $D=\|\alpha\| / 2$. Let us denote by $\Sigma_{\alpha, D}^{+}\left(\operatorname{resp} . \Sigma_{\alpha, D}^{-}\right)$the restriction of $\Sigma_{\alpha}$ to $\alpha((D,+\infty))(\operatorname{resp} . \alpha(-D,-\infty))$. Let us now define:

$$
U_{+}=\vec{n}\left(\Sigma_{\alpha, D}^{+}\right) \cup p_{+}, \quad U_{-}=\vec{n}\left(\Sigma_{\alpha, D}^{-}\right) \cup p_{-} .
$$

The mapping $\alpha$ sends the interior of the complement of $U_{-}$onto $U_{+}$. By Lemma 2.3.1:

$$
U_{ \pm} \subseteq \Omega_{\Delta_{k}(D, \pi / 2)}\left(p_{ \pm} ; \alpha(0)\right) .
$$

Since $\Delta_{k}(D, \pi / 2)<\pi / 2$, by continuity, there exists $\in \in \mathbb{R}^{+}$and $\theta \in(0, \pi)$ such that, for all $t \in(-\epsilon, \epsilon)$ :

$$
\Omega_{2 \theta}\left(p_{0} ; \alpha(t)\right) \cap U_{ \pm}=\emptyset .
$$

Let $U$ be a neighbourhood of $p_{0}$ in $M \cup \partial_{\infty} M$ such that:

$$
\Delta_{k}(d(\gamma, U), \pi-\theta)<\Delta_{k}(B / 2, \pi / 2) .
$$

Let $V$ be a neighbourhood of $p_{0}$ in $\partial_{\infty} U$ as in Lemma 2.3.2. Let $q_{ \pm}$be points in $V$ and let $\beta$ be the unique geodesic in $M$ such that $\beta( \pm \infty)=q_{ \pm}$.

Let $\gamma: \mathbb{R} \rightarrow M$ be the unique geodesic meeting both $\alpha$ and $\beta$ at right angles. By restricting $U$ if necessary, we may suppose that $\gamma(0) \in \alpha((-\epsilon, \epsilon))$ and that:

$$
\gamma(+\infty) \in \Omega_{\theta}\left(p_{0} ; \gamma(0)\right) \text {. }
$$

We may suppose that $\beta$ meets $\gamma$ at $\beta(0)$. Let us define $\Sigma_{\beta, B / 2}^{ \pm}$as for $\Sigma_{\alpha, D}^{ \pm}$and:

$$
V_{ \pm}=\vec{n}\left(\Sigma_{\beta, B / 2}^{ \pm}\right) \cup\left\{q_{ \pm}\right\} .
$$

The mapping $\beta$ sends the interior of the complement of $V_{-}$onto $V_{+}$. Moreover:

$$
\Omega_{\Delta_{k}(B / 2, \pi / 2)}(\gamma(-\infty) ; \beta(0)) \cap V_{ \pm}=\emptyset .
$$

However:

$$
\Omega_{\theta}(\gamma(+\infty) ; \gamma(0)) \cap U_{ \pm}=\emptyset
$$

Consequently:

$$
\begin{aligned}
& U_{ \pm} \subseteq \Omega_{\pi-\theta}(\gamma(-\infty) ; \gamma(0)) \\
\Rightarrow & U_{ \pm} \subseteq \Omega_{\Delta_{k}(B / 2, \pi / 2)}(\gamma(-\infty) ; \beta(0)) .
\end{aligned}
$$


The sets $U_{ \pm}$and $V_{ \pm}$are thus disjoint, and the result now follows by the classical "Ping-Pong" Lemma.

This yields the following useful corollary:

Corollary 2.4.2 Let $k>0$ be a real number and let $M$ be a Hadamard manifold with curvature less than $-k$. Let $\Gamma$ be a subgroup of $\operatorname{Isom}(M)$ which only contains hyperbolic elements (and the identity). Let $\gamma$ be a hyperbolic element of $\Gamma$ and let $p_{ \pm}$be the attractive and repulsive fixed points of $\gamma$ respectively. If $\alpha, \beta$ are elements of $\Gamma$ having no fixed points in common with $\gamma$, then there exists $N>0$ such that, for $|n| \geqslant N$, the subgroup of $\operatorname{Isom}(M)$ generated by $\gamma^{n} \alpha \gamma^{-n}$ and by $\beta$ is a Schottky group.

Proof Since $\alpha$ does not fix $p_{-}$, we find that the fixed points of $\gamma^{-n} \alpha \gamma^{n}$ tend towards $p_{+}$as $n$ tends towards $+\infty$. By hypothesis, $p_{+}$is not a fixed point of $\beta$. Moreover, $\alpha$ is not equal to the identity and is thus hyperbolic. Next, we see that:

$$
\left\|\gamma^{-n} \alpha \gamma^{n}\right\|=\|\alpha\|>0 .
$$

By the preceding lemma, there exists $N \geqslant 0$ such that, for $n \geqslant N$, the subgroup of $\operatorname{Isom}(M)$ generated by $\gamma^{-n} \alpha \gamma^{n}$ and $\beta$ is a Schottky group. A similar reasoning permits us to prove the result for $n$ negative, and the result now follows.

2.5 Proofs of main results of this section

We are now in a position to prove the propositions stated in the introduction to this section:

\section{Proof of Proposition 2.1.2}

(1) This holds for all $k$.

(2) This holds for all but at most one value of $k$. There thus exists $K$ such that, for $|k| \geqslant K$, the mapping $\delta_{k}$ is hyperbolic.

(3) For $|k| \geqslant K$, the mapping $\delta_{k}^{n} \alpha$ is hyperbolic for all but at most one value of $n$. Suppose that there exists $n \neq n^{\prime}$ such that $\delta_{k}^{n} \alpha$ and $\delta_{k}^{n^{\prime}} \alpha$ have the same fixed points as $\beta \alpha^{k}$. The mapping $\delta_{k}$ thus has the same fixed points as $\beta \alpha^{k}$. Consequently, so does $\alpha$, and thus also $\beta$. This is absurd.

(4) For $|k| \geqslant K$, this holds for all but at most one value of $n$, and the result now follows.

Proof of Proposition 2.1.3 We begin by showing that, after exchanging $\xi$ and $\eta$ if necessary, there exists $K \in \mathbb{N}$ such that for $|k| \geqslant K$, the mapping $\delta_{k}=\eta \beta \alpha^{k}$ has no fixed point in common with $\xi$. We suppose the contrary in order to obtain a contradiction. Suppose that there exists $k \neq k^{\prime}$ such that $\eta \beta \alpha^{k}$ and $\eta \beta \alpha^{k^{\prime}}$ both share the same fixed points as $\xi$. Suppose, moreover, that $\xi \beta \alpha^{k}$ and $\xi \beta \alpha^{k^{\prime}}$ both share the same fixed points as $\eta$. From the first condition, we conclude that $\alpha$ has the same fixed points as $\xi$, and from the second we conclude that $\alpha$ has the same fixed points as $\eta$. It therefore follows that both $\alpha$ and $\beta$ have the same fixed points, which is absurd. The result now follows.

We now suppose that there exists $k \neq k^{\prime}$ such that $\delta_{k}$ and $\delta_{k^{\prime}}$ have the same fixed points as $\eta$. It follows that $\alpha$ does too, and thus so does $\beta$, which is absurd. Consequently, there exists $K$ such that for $|k| \geqslant K$ :

$$
\operatorname{Fix}\left(\delta_{k}\right) \cap \operatorname{Fix}(\eta)=\emptyset .
$$


In particular, $\delta_{k}$ is hyperbolic. Moreover, we may assume that, for all $|k| \geqslant K$ :

$$
\operatorname{Fix}\left(\delta_{k}\right) \cap \operatorname{Fix}(\xi)=\emptyset .
$$

It follows by corollary 2.4.2 that there exists $N_{1}(k)$ such that, for $|n| \geqslant N_{1}(k)$ the group $\left\langle\delta_{k}^{n} \xi \delta_{k}^{-n}, \eta\right\rangle$ is a Schottky group. The result now follows.

Proof of Proposition 2.1.4 If $\sigma=\mathrm{Id}$, then $J_{k}=\alpha \beta$ for all $k$. This is necessarily hyperbolic, and the result follows in this case. Now suppose that $\sigma$ is hyperbolic. Suppose that there exists $k \neq k^{\prime}$ such that $J_{k}=J_{k^{\prime}}=\mathrm{Id}$. Then:

$$
\mathrm{Id}=J_{k} J_{k^{\prime}}^{-1} \text {. }
$$

If we denote $n=k-k^{\prime}$, we obtain:

$$
\begin{aligned}
& \sigma^{n} \alpha \sigma^{-n}=\alpha \\
& \Rightarrow \operatorname{Fix}(\alpha) \cdot \sigma^{-n}=\operatorname{Fix}(\alpha) \\
& \Rightarrow \operatorname{Fix}(\alpha)=\operatorname{Fix}(\sigma) .
\end{aligned}
$$

Likewise, $\operatorname{Fix}(\beta)=\operatorname{Fix}(\sigma)=\operatorname{Fix}(\alpha)$, which is absurd. The result now follows.

Proof of Proposition 2.1.5

(1) Since $\langle\xi, \eta\rangle$ is a Schottky group:

$$
\begin{aligned}
& \alpha \beta \alpha^{-1} \beta^{-1}=\eta \xi \neq \xi \\
& \Rightarrow \alpha \beta \alpha^{-1} \beta^{-1} \xi^{-1} \neq \mathrm{Id} \\
& \Rightarrow \beta \alpha^{-1} \beta^{-1} \xi^{-1} \alpha \neq \mathrm{Id} .
\end{aligned}
$$

(2) We suppose that there exists $k \neq k^{\prime}$ such that $\beta \alpha^{k}$ and $\beta \alpha^{k^{\prime}}$ share the same fixed points. It follows that $\alpha$, and thus also $\beta$, share the same fixed points as these mappings, which is absurd. Consequently, there exists $K_{1}$ such that, for $|k| \geqslant K_{1}$, the mapping $\gamma_{k}$ has no fixed points in common with $\xi$ or $\beta \alpha^{-1} \beta^{-1} \xi^{-1} \alpha$. By conjugation, for $|k| \geqslant K_{1}, \alpha \gamma_{k} \alpha^{-1}$ has no fixed point in common with $\alpha \beta \alpha^{-1} \beta^{-1} \xi^{-1}$. Moreover, for $|k| \geqslant K_{1}$, the mapping $\delta_{k}=\xi \gamma_{k}$ does not have any fixed points in common with $\gamma_{k}$.

(3) If $\operatorname{Fix}\left(\delta_{k}\right)=\operatorname{Fix}(\eta)$, then $\delta_{k}$ commutes with $\eta$. Thus, $\left\langle\xi, \delta_{k}^{-n} \eta \delta_{k}^{n}\right\rangle=\langle\xi, \eta\rangle$ is a Schottky group for all $n$. Otherwise, $\operatorname{Fix}\left(\delta_{k}\right) \cap \operatorname{Fix}(\eta)=\emptyset$. Since $\operatorname{Fix}\left(\delta_{k}\right) \cap \operatorname{Fix}(\xi) \neq \emptyset$, it follows by corollary 2.4.2 that there exists $N_{1}(k)$ such that, for $|n| \geqslant N_{1}(k)$, the group $\left\langle\xi, \delta_{k}^{-n} \eta \delta_{k}^{n}\right\rangle$ is a Schottky group.

(4) If $\delta_{k}$ shares the same fixed points as $\alpha \gamma_{k} \alpha^{-1}$, then so does $\alpha \beta \alpha^{-1} \beta^{-1} \xi^{-1}$, which is absurd by (2). If $\delta_{k}$ shares the same fixed points as $\gamma_{k}$, then so does $\xi$, which is also absurd by (2). It thus follows by corollary 2.4.2 that there exists $N_{2}(k) \geqslant N_{1}(k)$ such that, for $|n| \geqslant N_{2}(k)$, the group $\left\langle\alpha \gamma_{k} \alpha^{-1}, \delta_{k}^{n-1} \gamma_{k}^{-1} \delta_{k}^{n}\right\rangle$ is a Schottky group, and the result now follows by conjugation with $\alpha$.

\section{The pants decomposition}

\subsection{Introduction}

Throughout this section $(M, Q)$ denotes a pointed, compact, Riemannian manifold of strictly negative sectional curvature, $(\tilde{M}, \tilde{Q})$ denotes its universal cover, $(\Sigma, P)$ denotes a pointed, compact surface of genus at least 2 and $\theta: \pi_{1}(\Sigma, P) \rightarrow \pi_{1}(M, Q)$ denotes a representation 
of $\pi_{1}(\Sigma, P)$ in $\pi_{1}(M, Q)$. In [4], Gallo, Kapovich and Marden obtain a pants decomposition of $\Sigma$ such that the $\theta$-image of the fundamental group of each pant is a Schottky group. In this section, we will prove the following lemma, which is the generalisation to our framework of this part of Gallo, Kapovich and Marden's result:

Lemma 3.1.1 Let $(M, Q)$ be a pointed, compact, three dimensional Riemannian manifold of strictly negative sectional curvature. Let $(\Sigma, \theta)$ be a pointed, orientable, compact surface of hyperbolic type (i.e. of genus greater than or equal to 2 ) with holonomy in $\pi_{1}(M, Q)$. Then there exist bound, marked pants $\left(T_{i}, \theta_{i}, \beta_{i}\right)_{1 \leqslant i \leqslant 2 g-2}$ with holonomy in $\pi_{1}(M, Q)$ such that:

(i) for all $i$, the image of $\theta_{i}$ is a Schottky group, and

(ii)

$$
(\Sigma, \theta) \cong \stackrel{Ð}{g}_{i=1}^{2 g-2}\left(\bigcup_{i=1}^{\bigcup}\left(T_{i}, \theta_{i}, \beta_{i}\right)\right) .
$$

The terms and terminology used in the statement of this lemma will be explained in the second and third parts of this section.

The proof of this lemma is an immediate consequence of Propositions 3.4.1, 3.5.1 and 3.6.1. In Proposition 3.4.1, the surface is cut open to yield a surface of genus 1 and $2 g-2$ boundary components. Using an induction argument driven by Proposition 3.5.1, we successively remove pants until we are left with a surface of genus 1 and 2 boundary components. Here, a more subtle reasoning, provided in proposition 3.6.1 allows us to carry out the final cut thus proving the lemma.

In the second part of this section, we define the notion of a marked surface with holonomy in a group $G$. In the third part, we define the notion of a binding, which is required as a bookkeeping measure in order to recover correctly the fundamental group after the surface has been cut up into pants. In the remaining parts, we successively decompose the surface $\Sigma$, proving Propositions 3.4.1, 3.5.1 and 3.6.1.

\subsection{Marked surfaces and handles}

The group $\pi_{1}(M, Q)$ may be considered as a subgroup of the group of isometries of $\tilde{M}$. Since $M$ is compact, every element of $\pi_{1}(M, Q) \backslash\{\mathrm{Id}\}$, viewed as an isometry of $\tilde{M}$, is hyperbolic in the sense of section 2.1. If $a$ and $b$ are two points in $\partial_{\infty} \tilde{M}$, and if $\gamma \in \pi_{1}(M, Q)$, then $\gamma$ cannot send $a$ to $b$ and $b$ to $a$, since, otherwise, it would be elliptic. Moreover, we recall that the fixed point sets of two elements of $\pi_{1}(M, Q)$ are either disjoint or equal.

Let $G$ be a group. We define a marked surface with holonomy in $G$ to be a triplet of:

(i) a pointed compact surface with boundary $\left(\Sigma, \partial \Sigma, P_{0}\right)$, with $\partial \Sigma$ oriented such that the interior of $\Sigma$ lies to its left,

(ii) for every connected component $C_{\alpha}$ of $\partial \Sigma$, a point $Q_{\alpha} \in C_{\alpha}$, which is the base point of $C_{\alpha}$, and

(iii) a homomorphism $\theta: \pi_{1}\left(\Sigma, P_{0}\right) \rightarrow G$.

By slight abuse of notation, we will refer to the marked surface with holonomy simply by $(\Sigma, \theta)$. Every closed curve in $\Sigma$ defines canonically a conjugacy class in $\pi_{1}\left(\Sigma, P_{0}\right)$. For such a closed curve, $a$, we denote the corresponding conjugacy class by $[a]$. The $\theta$-image of $[a]$ defines a conjugacy class in $G$ which we denote by $\theta[a]$. If $G$ is a subgroup of $\pi_{1}(M, Q)$, and if one element in a given conjugacy class of $G$ is hyperbolic, then all elements in that 
conjugacy class are hyperbolic, and the class is said to be hyperbolic. A closed curve $a$ in $(\Sigma, \theta)$ is then said to be hyperbolic if and only if $\theta[a]$ is hyperbolic.

Every pair $(a, b)$ of simple closed curves in $\Sigma$ with the same base point generates a conjugacy class of pairs in $\pi_{1}\left(\Sigma, P_{0}\right)$. Indeed, with $x$ a curve joining $P_{0}$ to the common base point of $a$ and $b$, we define:

$$
(a, b)_{x}=\left(x^{-1} a x, x^{-1} b x\right) \in \pi_{1}\left(\Sigma, P_{0}\right) \times \pi_{1}\left(\Sigma, P_{0}\right) .
$$

The conjugacy class of this pair is independent of $x$, and we denote it by $[a, b]$.

The $\theta$-image of the class $[a, b]$ defines a conjugacy class of pairs in $G$ which we denote by $\theta[a, b]$. Every element of $\theta[a, b]$ generates a subgroup of $G$, and $\theta[a, b]$ thus defines a conjugacy class of subgroups of $G$ which we denote by $\langle\theta[a, b]\rangle$. If $G$ is a subgroup of $\pi_{1}(M, Q)$, a subgroup in a given conjugacy class in $G$ is a Schottky group if and only if every group in that class is too, and the class is said to be Schottky. The pair $(a, b)$ is said to be Schottky if and only if $\langle\theta[a, b]\rangle$ is Schottky. We define a non-elementary pair and a non-elementary class in the same manner.

Let $(\Sigma, \theta)$ be an (oriented) marked surface with holonomy in $G$. A marked handle in $(\Sigma, \theta)$ is a pair $(a, b)$ of non-dividing (oriented) simple closed curves in $(\Sigma, \theta)$ such that $a$ and $b$ only intersect at their common base point, and $b$ crosses $a$ from right to left. When $G$ is a subgroup of the group $\pi_{1}(M, Q)$, we say that $(a, b)$ is a hyperbolic handle if and only if both $a$ and $b$ are hyperbolic. We say that the handle $(a, b)$ is non-elementary or Schottky if and only if, viewed as a pair, it is non-elementary or Schottky respectively.

Finally, for $(\Sigma, \theta)$ a marked surface with boundary with holonomy in $G$, and for a given family, $\left(Q_{i}\right)_{i \in F}$ of points in $(\Sigma, \theta)$, we define a sash to be a set of simple curves $\left(\gamma_{i}\right)_{i \in F}$ in $(\Sigma, \theta)$ indexed by the same set as $\left(Q_{i}\right)_{i \in F}$ such that:

(i) for every $i, \gamma_{i}$ goes from $P_{0}$ to $Q_{i}$,

(ii) for every $i \neq j, \gamma_{i}$ only intersects $\gamma_{j}$ at $P_{0}$,

(iii) for every $i, \gamma_{i}$ can only intersect $\partial \Sigma$ at $Q_{i}$.

Sashes permit us to identify objects in $\Sigma$ (curves, marked handles, etc.) explicitly with elements or pairs of elements of $\pi_{1}\left(\Sigma, P_{0}\right)$.

When we define many such geometric structures over $(\Sigma, \theta)$, we will assume them to be disjoint, except, possibly, at their end or base points.

\subsection{Bindings}

Let $G$ be a group. Let $(\Sigma, \theta)$ be a marked surface with holonomy in $G$. Suppose that $\Sigma$ has $n$ boundary components, and let $\left(C_{i}, Q_{i}\right)_{1 \leqslant i \leqslant n}$ be the pointed oriented boundary components of $\Sigma$. For every $i$, denote the set of all homotopy classes of curves in $\Sigma$ going from $P_{0}$ to $Q_{i}$ by $\pi_{1}\left(\Sigma, P_{0}, Q_{i}\right)$. A binding of $(\Sigma, \theta)$ is a set of mappings $\left(\beta_{i}\right)_{1 \leqslant i \leqslant n}$ such that:

(i) for every $i, \beta_{i}$ maps $\pi_{1}\left(\Sigma, P_{0}, Q_{i}\right)$ into $G$, and

(ii) for every $i, \beta_{i}$ is equivariant under $\theta$ with respect to the canonical right action of $\pi_{1}\left(\Sigma, P_{0}\right)$ on $\pi_{1}\left(\Sigma, P_{0}, Q_{i}\right)$. In other words, for all $\xi \in \pi_{1}\left(\Sigma, P_{0}, Q_{i}\right)$, and for all $\eta \in \pi_{1}\left(\Sigma, P_{0}\right)$ :

$$
\beta_{i}(\xi \eta)=\beta_{i}(\xi) \theta(\eta) .
$$

In particular, since $\pi_{1}\left(\Sigma, P_{0}\right)$ acts simply transitively on $\pi_{1}\left(\Sigma, P_{0}, Q_{i}\right)$, condition (ii) ensures that a binding $\beta$ is uniquely determined by its action on a single element of $\pi_{1}\left(\Sigma, P_{0}, Q_{i}\right)$.

We will denote the binding by $\beta$, and, for every $i$, we will write $\beta$ instead of $\beta_{i}$ when there is no danger of ambiguity. A marked surface $(\Sigma, \theta)$ carrying a binding, $\beta$, will be said to be 
bound. We will denote the bound marked surface with holonomy in $G$ by $(\Sigma, \theta, \beta)$. For any oriented, pointed boundary component $(C, Q)$ of $\Sigma$, we define the element $\beta(C) \in G$ such that, for all $\xi \in \pi_{1}\left(\Sigma, P_{0}, Q\right)$ :

$$
\beta(C)=\beta(\xi) \theta\left(\xi^{-1} C \xi\right) \beta(\xi)^{-1} .
$$

Let $(\Sigma, \theta, \beta)$ and $\left(\Sigma^{\prime}, \theta^{\prime}, \beta^{\prime}\right)$ be bound marked surfaces with holonomy in $G$. Let $(C, Q)$ and $\left(C^{\prime}, Q^{\prime}\right)$ be oriented, pointed boundary components of $\Sigma$ and $\Sigma^{\prime}$ respectively. Let $\varphi$ : $(C, Q) \rightarrow\left(C^{\prime-1}, Q^{\prime}\right)$ be an orientation reversing homeomorphism. Let $\Sigma \cup_{\varphi} \Sigma^{\prime}$ be the surface obtained by joining $\Sigma$ to $\Sigma^{\prime}$ along $\varphi$. We identify all objects (points, curves, etc.) in $\Sigma$ and $\Sigma^{\prime}$ with the corresponding objects in $\Sigma \cup_{\varphi} \Sigma^{\prime}$ and vice-versa. We take the base point of $\Sigma \cup_{\varphi} \Sigma^{\prime}$ to be that of $\Sigma$.

We say that $(\theta, \beta)$ and $\left(\theta^{\prime}, \beta^{\prime}\right)$ may be joined along $\varphi$ if and only if $\beta(C)^{-1}=\beta^{\prime}\left(C^{\prime}\right)$. In this case, we define $\theta \cup_{\varphi} \theta^{\prime}$, the joined union of $\theta$ and $\theta^{\prime}$ along $\varphi$, such that:

(i) for all $x \in \pi_{1}\left(\Sigma, P_{0}\right)$ :

$$
\left(\theta \cup_{\varphi} \theta^{\prime}\right)(x)=\theta(x)
$$

and

(ii) for all $x \in \pi_{1}\left(\Sigma^{\prime}, P_{0}^{\prime}\right), \xi \in \pi_{1}\left(\Sigma, P_{0}, Q\right)$ and $\eta \in \pi_{1}\left(\Sigma^{\prime}, P_{0}^{\prime}, Q^{\prime}\right)$ :

$$
\left(\theta \cup_{\varphi} \theta^{\prime}\right)\left(\xi^{-1} \eta x \eta^{-1} \xi\right)=\beta(\xi)^{-1} \beta^{\prime}(\eta) \theta^{\prime}(x) \beta^{\prime}(\eta)^{-1} \beta(\xi) .
$$

The join condition on $(\theta, \beta)$ and $\left(\theta^{\prime}, \beta^{\prime}\right)$ ensures that $\theta \cup_{\varphi} \theta^{\prime}$ is a well defined homomorphism from $\pi_{1}\left(\Sigma \cup_{\varphi} \Sigma^{\prime}, P_{0}\right)$ into $G$. We may also define $\beta \cup_{\varphi} \beta^{\prime}$, the joined union of $\beta$ and $\beta^{\prime}$ along $\varphi$ in the same manner, with the join condition again ensuring that $\beta \cup_{\varphi} \beta^{\prime}$ is well defined. In the sequel, the homeomorphism $\varphi$ will be suppressed, and we thus denote:

$$
(\Sigma, \theta, \beta) \cup\left(\Sigma^{\prime}, \theta^{\prime}, \beta^{\prime}\right)=\left(\Sigma \cup \Sigma^{\prime}, \theta \cup \theta^{\prime}, \beta \cup \beta^{\prime}\right) .
$$

In a similar manner, we may join a bound marked surface to itself along an orientation reversing homeomorphism between two boundary components. We denote the join of $(\Sigma, \theta, \beta)$ to itself by:

$$
\bigcirc(\Sigma, \theta, \beta)=(\bigcirc \Sigma, \bigcirc \theta, \bigcirc \beta) .
$$

For surfaces with many boundary components, this process may be iterated, and we will refer to the $n$-fold join of $(\Sigma, \theta, \beta)$ to itself by:

$$
\bigcirc^{n}(\Sigma, \theta, \beta) \text {. }
$$

\subsection{Slicing open the surface}

In this subsection, we will prove the following result:

Proposition 3.4.1 Let $\left(M, Q_{0}\right)$ be a pointed, compact, three dimensional manifold of strictly negative sectional curvature. Let $(\Sigma, \theta)$ be a compact surface without boundary of genus $g \geqslant 2$ with holonomy $\theta$ in $\pi_{1}\left(M, Q_{0}\right)$.

If the image of $\theta$ is non-elementary, then there exists $\left(\Sigma_{2 g-2}, \theta_{2 g-2}, \beta_{2 g-2}\right)$, a bound marked surface with holonomy in $\pi_{1}\left(M, Q_{0}\right)$ of genus 1 with a non-elementary marked handle $(a, b)$ and $2 g-2$ boundary components, such that:

(i) every connected component of $\partial \Sigma_{2 g-2}$ is hyperbolic, and 


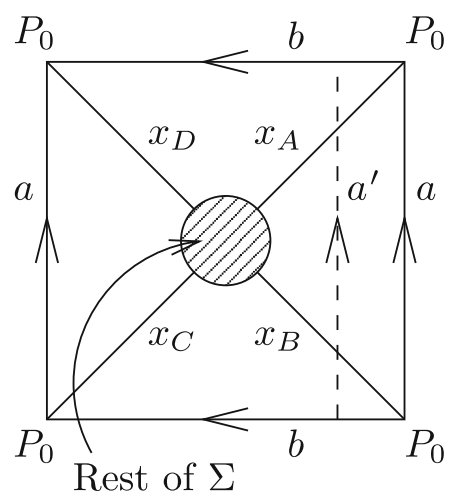

Fig. 1

(ii) $(\Sigma, \theta)$ is homeomorphic to $\bigcirc^{g-1}\left(\Sigma_{2 g-2}, \theta_{2 g-2}, \beta_{2 g-2}\right)$.

If $\Sigma$ is a closed surface of genus $g \geqslant 2$, then we define a canonical basis of $\Sigma$ to be an n-tuple $\left[\left(a_{1}, b_{1}\right), \ldots,\left(a_{n}, b_{n}\right)\right]$ of pairs of simple closed curves in $\Sigma$ which correspond to handles in $\Sigma$, such that:

(i) for all $i \neq j$ the pairs $\left(a_{i}, b_{i}\right)$ and $\left(a_{j}, b_{j}\right)$ are disjoint,

(ii) for all $i$, the curves $a_{i}$ and $b_{i}$ have a common base point, which is their only shared point and which we denote by $Q_{i}$, and

(iii) for all $i, b_{i}$ crosses $a_{i}$ from right to left.

We have the following result:

Proposition 3.4.2 Let $\left(M, Q_{0}\right)$ be a pointed compact three dimensional Hadamard manifold of strictly negative sectional curvature. Let $(\Sigma, \theta)$ be a compact surface without boundary of genus $g \geqslant 2$ with holonomy in $\pi_{1}\left(M, Q_{0}\right)$. Let $\left[\left(a_{1}, b_{1}\right), \ldots,\left(a_{n}, b_{n}\right)\right]$ be a canonical basis of $\Sigma$.

If $\theta$ is non-elementary, then there exists a homeomorphism $\Phi$ of $\Sigma$ such that $\Phi_{*}\left(a_{1}, b_{1}\right)$ is non-elementary.

Proof Since the image of $\theta$ is non-elementary, after composing with a homeomorphism of $\Sigma$ which permutes the generators of $\pi_{1}(\Sigma)$, we may suppose that the class $\theta\left(a_{1}\right)$ is hyperbolic. For all $i$, let $Q_{i}$ be the common base point of $a_{i}$ and $b_{i}$. Let $\left(c_{i}\right)_{1 \leqslant i \leqslant g}$ be a sash of $\left(Q_{i}\right)_{1 \leqslant i \leqslant g}$. Let us define $a=c_{1}^{-1} a_{1} c_{1}$ and $b=c_{1}^{-1} b_{1} c_{1}$. Let us next define $\alpha=\theta(a)$ and $\beta=\theta(b)$. There are two cases:

(1) $\beta$ does not have any fixed points in common with $\alpha$. In this case, we take $\Phi=\operatorname{Id}$ and we obtain the desired result.

(2) $\beta$ has two fixed points in common with $\alpha$ (in particular, $\beta$ could be the identity). Since $\Gamma$ is non-elementary, there exists $x \in\left\{c_{i}^{-1} a_{i} c_{i}, c_{i}^{-1} b_{i} c_{i} \mid 2 \leqslant i \leqslant n\right\}$ such that $\xi=\theta(x)$ does not have any fixed points in common with $\alpha$.

We may orient $x$ such that $c=a x$ is freely homotopic to a simple closed curve. Let $T$ be the Dehn twist about $c$. In order to simplify the discussion, we identify $Q_{1}$ with $P_{0}$. Figure 1 shows the handle of $\Sigma$ defined by $(a, b)$ opened up by cutting along $a$ and $b$.

The curve $x$ terminates at $P_{0}$ without crossing either $a$ or $b$. This corresponds to one of the four topologically distinct configurations $x_{A}, x_{B}, x_{C}$ or $x_{D}$ shown in Fig. 1. By defining $c$ 
using a slightly displaced copy of $a$, we may ensure that $c$ never crosses $a$. If $x$ is in one of the configurations $A$ or $B$, this would correspond to taking a copy of $a$ displaced to its left, and if $x$ is in one of the configurations $C$ or $D$, then this would correspond to taking a copy of $a$ displaced to its right, as shown in Fig. 1. If we choose $x$ in configuration $A$, then the Dehn twist, $T$, about $c$ satisfies:

$$
T_{*} a=a, \quad T_{*} b=b a x .
$$

Now:

$$
\operatorname{Fix}(\beta \alpha \xi)=\operatorname{Fix}(\alpha) \Leftrightarrow \operatorname{Fix}(\xi)=\operatorname{Fix}(\alpha) .
$$

It thus follows that $\operatorname{Fix}(\beta \alpha \xi) \cap \operatorname{Fix}(\alpha)=\emptyset$. Defining $\Psi=T$, we obtain the desired homeomorphism.

We next have:

Proposition 3.4.3 Let $\left(M, Q_{0}\right)$ be a pointed compact three dimensional Hadamard manifold of strictly negative sectional curvature. Let $(\Sigma, \theta)$ be a compact surface without boundary of genus $g \geqslant 2$ with holonomy in $\pi_{1}\left(M, Q_{0}\right)$. Let $\left[\left(a_{1}, b_{1}\right), \ldots,\left(a_{g}, b_{g}\right)\right]$ be a canonical basis of $\Sigma$.

If $\left(a_{1}, b_{1}\right)$ is non-elementary, then, for all $i$ different to 1 , there exists a homeomorphism $\Psi$ of $\Sigma$ such that:

(1) $\Psi_{*}\left(a_{1}, b_{1}\right)$ is non-elementary,

(2) $\Psi_{*} a_{i}$ and $\Psi_{*} b_{i}$ are hyperbolic, and

(3) for all $j$ different from 1 and $i$, the mapping $\Psi_{*}$ acts on $a_{j}$ and $b_{j}$ by conjugation.

Using induction, we thus obtain the following result:

Corollary 3.4.4 With the same hypotheses as in Proposition 3.4.3, there exists a homeomorphism $\Psi$ such that:

(1) $\Psi_{*}\left(a_{1}, b_{1}\right)$ is non-elementary, and

(2) for all $i$ different from $1, \Psi_{*} a_{i}$ and $\Psi_{*} b_{i}$ are hyperbolic.

Proof of Proposition 3.4.3 For all $i$, let $Q_{i}$ be the common base point of $a_{i}$ and $b_{i}$. Let $\left(c_{i}\right)_{1 \leqslant i \leqslant g}$ be a sash of $\left(Q_{i}\right)_{1 \leqslant i \leqslant g}$. As before, we denote $a=c_{1}^{-1} a_{1} c_{1}$ and $b=c_{1}^{-1} b_{1} c_{1}$. We now define $\alpha=\theta(a)$, and $\beta=\theta(b)$. For a given $i$ different to 1 , we denote $x=c_{i}^{-1} a_{i} c_{i}$, and $y=c_{i}^{-1} b_{i} c_{i}$, and we define $\xi=\theta(x)$ and $\eta=\theta(y)$.

We orient $y$ such that $C=y b$ is freely homotopic to a simple closed curve. We denote $d_{0}=y b$. Let $T_{0}$ be a Dehn twist about $C$. The curves $c_{1}$ and $c_{i}$ may be chosen such that:

$$
\begin{array}{ll}
\left(T_{0}\right)_{*} a=d_{0} a, & \left(T_{0}\right)_{*} b=b, \\
\left(T_{0}\right)_{*} x=d_{0} x, & \left(T_{0}\right)_{*} y=y .
\end{array}
$$

Since $\left(T_{0}\right)_{*}$ leaves $d_{0}$ invariant, we obtain, for all $n$ :

$$
\begin{array}{ll}
\left(T_{0}^{n}\right)_{*} a=d_{0}^{n} a, & \left(T_{0}^{n}\right)_{*} b=b, \\
\left(T_{0}^{n}\right)_{*} x=d_{0}^{n} x, & \left(T_{0}^{n}\right)_{*} y=y .
\end{array}
$$

Moreover, for all $j$ different from 1 and $i$, since $d_{0}$ only intersects $c_{j}$ near $P_{0}$, it follows that $\left(T_{0}\right)_{*}$ acts on $\left(a_{j}, b_{j}\right)$ by conjugation. Let $T_{a}$ be the Dehn twist about $a$ such that, for all $k$ :

$$
\begin{aligned}
& \left(T_{a}^{k}\right)_{*} a=a, \quad\left(T_{a}^{k}\right)_{*} b=b a^{k} \\
& \left(T_{a}^{k}\right)_{*} x=x, \quad\left(T_{a}^{k}\right)_{*} y=y .
\end{aligned}
$$


For all $j$ different to 1 , since $a$ stays away from $\left(a_{j}, b_{j}\right),\left(T_{a}\right)_{*}$ has no effect on this pair. We define $\Psi_{k, n}: \Sigma \rightarrow \Sigma$ by:

$$
\Psi_{k, n}=T_{a}^{k} T_{0}^{n} T_{a}^{-k} .
$$

If, for all $k$, we define $d_{k}$ by $d_{k}=y b a^{k}$, then we obtain:

$$
\begin{array}{ll}
\left(\Psi_{k, n}\right)_{*} a=d_{k}^{n} a, & \left(\Psi_{k, n}\right)_{*} b a^{k}=b a^{k}, \\
\left(\Psi_{k, n}\right)_{*} x=d_{k}^{n} x, & \left(\Psi_{k, n}\right)_{*} y=y .
\end{array}
$$

Where $d_{k}=y b a^{k}$. Moreover, for all $j$ different to 1 and $i$, the mapping $\Psi_{k, n}$ acts on $\left(a_{j}, b_{j}\right)$ by conjugation. We now choose $k$ and $n$ as in Lemma 2.1.2. If $\eta$ is hyperbolic, then, taking $\Psi=\Psi_{k, n}$, we obtain the desired result. Otherwise, $\eta=\mathrm{Id}$, and we may take a Dehn twist $T$ about $x$ such that $T y=x y$ and which leaves all the other curves invariant. We see that:

$$
\theta\left(\Psi_{k, n}\right)_{*} T_{*} y=\theta \Psi_{k, n_{*}} x y=\theta d_{k}^{n} x y=\delta_{k}^{n} \xi,
$$

and this is hyperbolic. We thus denote $\Psi=\Psi_{k, n} \circ T$ and we once again obtain the desired result.

The proof of Proposition 3.4.1 is now elementary:

Proof of Proposition 3.4.1 Let $\left[\left(a_{1}, b_{1}\right), \ldots,\left(a_{n}, b_{n}\right)\right]$ be a canonical basis of $\Sigma$. By Proposition 3.4.2 and Corollary 3.4.4, we may suppose that $\left(a_{1}, b_{1}\right)$ is a non-elementary marked handle and that, for all $2 \leqslant i \leqslant n, a_{i}$ and $b_{i}$ are hyperbolic.

We obtain $\Sigma_{2 g-2}$ by cutting $\Sigma$ along each $a_{i}$ for $2 \leqslant i \leqslant n$. We identify objects (points, curves etc.) in $\Sigma$ with the corresponding objects in $\Sigma_{2 g-2}$, and vice-versa. For each $i$, we denote by $C_{i,-}$ the copy of $a_{i}$ in $\Sigma_{2 g-2}$ from which $b_{i}$ leaves and by $C_{i,+}$ the copy of $a_{i}^{-1}$ in $\Sigma_{2 g-2}$ at which $b_{i}$ arrives, and we define $Q_{i, \pm}$ to be the copy of $Q_{i}$ lying in $C_{i, \pm}$. Let $\left(\gamma_{i}\right)_{2 \leqslant i \leqslant n}$ be a sash of $\left(Q_{i,-}\right)_{2 \leqslant i \leqslant n}$. We define the binding $\beta_{2 g-2}$ over $\Sigma_{2 g-2}$ such that, for all $i$ :

$$
\begin{aligned}
\beta_{2 g-2}\left(\gamma_{i}\right) & =\mathrm{Id}, \\
\beta_{2 g-2}\left(b_{i} \gamma_{i}\right) & =\theta\left(\gamma_{i}^{-1} b_{i} \gamma_{i}\right) .
\end{aligned}
$$

We may verify that this binding satisfies the appropriate join conditions and that:

$$
(\Sigma, \theta) \cong \bigcirc^{g-1}\left(\Sigma_{2 g-2}, \theta_{2 g-2}, \beta_{2 g-2}\right) .
$$

Finally, we define the marked handle $(a, b)$ in $\Sigma_{2 g-2}$ by:

$$
(a, b)=\left(a_{1}, b_{1}\right) .
$$

Since $\left(a_{1}, b_{1}\right)$ is hyperbolic, so is $(a, b)$, and the result now follows.

\subsection{Pruning}

In this subsection, we aim to prove the following result:

Proposition 3.5.1 Let $(M, Q)$ be a pointed, compact, three dimensional manifold of strictly negative sectional curvature. Let $\left(\Sigma_{2 g-2}, \theta_{2 g-2}, \beta_{2 g-2}\right)$ be a bound marked surface with holonomy in $\pi_{1}(M, Q)$ of genus 1 having $2 g-2$ boundary components, all of which are hyperbolic, and a non-elementary marked handle $(a, b)$. There exists: 
(1) a bound marked surface $\left(\Sigma_{2}, \theta_{2}, \beta_{2}\right)$ with holonomy in $\pi_{1}(M, Q)$, and

(2) $2 g-4$ bound, marked pants $\left(T_{i}, \varphi_{i}, \beta_{i}\right)_{1 \leqslant i \leqslant 2 g-4}$ with holonomy in $\pi_{1}(M, Q)$, such that:

$$
\left(\Sigma_{2 g-2}, \theta_{2 g-2}, \beta_{2 g-2}\right) \cong\left(\Sigma_{2}, \varphi_{2}, \beta_{2}\right) \underset{i=1}{2 g-4}\left(T_{i}, \varphi_{i}, \beta_{i}\right)
$$

and:

(1) $\left(\Sigma_{2}, \theta_{2}, \beta_{2}\right)$ has genus 1 , two hyperbolic boundary components and a non-elementary marked handle $\left(a^{\prime}, b^{\prime}\right)$, and

(2) for all $i$, the image of $\varphi_{i}$ in $\pi_{1}(M, Q)$ is a Schottky group.

We begin with the following proposition:

Proposition 3.5.2 Let $(M, Q)$ be a pointed compact three dimensional manifold of strictly negative sectional curvature. Let $\left(\Sigma_{n}, \theta_{n}, \beta_{n}\right)$ be a bound marked surface with holonomy in $\pi_{1}(M, Q)$ of genus 1 having $n$ hyperbolic boundary components and a non-elementary marked handle $\left(a_{1}, b_{1}\right)$.

Let $x_{1}$ and $y_{1}$ be two boundary components of $\Sigma_{n}$. Let $c$ be a simple curve joining $x_{1}$ to $y_{1}$ which is disjoint from $x_{1}, y_{1}, a_{1}$ and $b_{1}$ except possibly at its end points.

There exists a homeomorphism $\Psi:\left(\Sigma_{n}, \partial \Sigma_{n}, P_{0}\right) \rightarrow\left(\Sigma_{n}, \partial \Sigma_{n}, P_{0}\right)$ of $\Sigma_{n}$ such that:

(1) $\Psi_{*}\left(a_{1}, b_{1}\right)$ is non-elementary,

(2) if $z$ is a boundary component of $\Sigma$ different to $x_{1}$ and $y_{1}$, then $\Phi_{*}$ acts on $z$ by conjugation (thus, if $\theta(z)$ is hyperbolic, then $\theta \Phi_{*} z$ is hyperbolic), and

(3) $\Psi_{*}\left(x_{1}, c^{-1} y_{1} c\right)$ is Schottky (and thus, in particular, $x_{1} c^{-1} y_{1} c$ is hyperbolic).

Proof Let $Q_{0}$ be the common base point of $a_{1}$ and $b_{1}$. Let $Q_{x}$ and $Q_{y}$ be the base points of $x_{1}$ and $y_{1}$ resp. Let $\left(c_{0}, c_{x}\right)$ be a sash of $\left(Q_{0}, Q_{x}\right)$. We define:

$$
\begin{aligned}
& a=c_{0}^{-1} a_{1} c_{0}, \quad b=c_{0}^{-1} b_{1} c_{0}, \\
& x=c_{x}^{-1} x_{1} c_{x}, \quad y=\left(c c_{x}\right)^{-1} y_{1}\left(c c_{x}\right) .
\end{aligned}
$$

We denote $\alpha=\theta_{n}(a), \beta=\theta_{n}(b), \xi=\theta_{n}(x)$ and $\eta=\theta_{n}(y)$.

We may suppose that $b$ is oriented such that $C=y b$ is homotopic to a simple closed curve. we denote $d_{0}=y b$. Let $T_{0}$ be a Dehn twist about $C$. We may suppose that $c_{a}$ and $c_{x}$ are chosen such that:

$$
\begin{aligned}
& \left(T_{0}\right)_{*} a=d_{0} a, \quad\left(T_{0}\right)_{*} b=b, \\
& \left(T_{0}\right)_{*} x=d_{0} x d_{0}^{-1}, \quad\left(T_{0}\right)_{*} y=y .
\end{aligned}
$$

Since $\left(T_{0}\right)_{*}$ is a homomorphism which leaves $d_{0}$ invariant, we obtain, for all $n$ :

$$
\begin{aligned}
\left(T_{0}^{n}\right)_{*} a=d_{0}^{n} a, & \left(T_{0}^{n}\right)_{*} b=b, \\
\left(T_{0}^{n}\right)_{*} x=d_{0}^{n} x d_{0}^{-n}, & \left(T_{0}^{n}\right)_{*} y=y .
\end{aligned}
$$

We choose a Dehn twist $T_{a}$ about $a$ such that, for all $k$ :

$$
\begin{aligned}
& \left(T_{a}^{k}\right)_{*} a=a, \quad\left(T_{a}^{k}\right)_{*} b=b a^{k}, \\
& \left(T_{a}^{k}\right)_{*} x=x, \quad\left(T_{a}^{k}\right)_{*} y=y .
\end{aligned}
$$


We define $\Psi_{k, n}: \Sigma_{2 n-2} \rightarrow \Sigma_{2 n-2}$ by:

$$
\Psi_{k, n}=T_{a}^{k} T_{0}^{n} T_{a}^{-k}
$$

Denoting $d_{k}=y b a^{k}$, we obtain:

$$
\begin{array}{r}
\left(\Psi_{k, n}\right)_{*} a=d_{k}^{n} a, \quad\left(\Psi_{k, n}\right)_{*} b a^{k}=b a^{k}, \\
\left(\Psi_{k, n}\right)_{*} x=d_{k}^{n} x d_{k}^{-n}, \quad\left(\Psi_{k, n}\right)_{*} y=y .
\end{array}
$$

We choose $k$ and $n$ as in Lemmata 2.1.2 and 2.1.3, and we denote $\Psi=\Psi_{k, n}$. The first result follows from the first and third conclusions of Lemma 2.1.2. Since $\Psi$ is a product of Dehn twists, it acts on paths corresponding to boundary components by conjugation. The second result now follows. The third result follows from Lemma 2.1.3.

This yields:

Corollary 3.5.3 With the same hypotheses as in Proposition 3.5.2, there exists:

(i) a bound marked pant $(T, \theta, \beta)$ with holonomy in $\pi_{1}(M, Q)$, and

(ii) a bound marked surface $\left(\Sigma_{n-1}, \theta_{n-1}, \beta_{n-1}\right)$ with holonomy in $\pi_{1}(M, Q)$, such that:

$$
\left(\Sigma_{n}, \theta_{n}, \beta_{n}\right) \cong\left(\Sigma_{n-1}, \theta_{n-1}, \beta_{n-1}\right) \cup(T, \theta, \beta),
$$

and,

(i) ( $\left.\Sigma_{n-1}, \theta_{n-1}, \beta_{n-1}\right)$ has genus $1, n-1$ hyperbolic boundary components, and a nonelementary marked handle $\left(a^{\prime}, b^{\prime}\right)$,

(ii) the image of $\theta$ in $\pi_{1}(M, Q)$ is a Schottky group.

Proof We may suppose that $(\Sigma, \theta)$ satisfies the conclusions of Proposition 3.5.2. Cutting $\Sigma_{n}$ along a curve freely homotopic to $x_{1} c^{-1} y_{1} c$ which does not intersect either $a$ or $b$, we obtain the desired result as in the proof of Proposition 3.4.1.

We may now prove Proposition 3.5.1:

Proof of Proposition 3.5.1 This follows directly from induction and Corollary 3.5.3.

3.6 Untying the root

In this subsection, we aim to prove the following result:

Proposition 3.6.1 Let $(M, Q)$ be a pointed compact three dimensional manifold of strictly negative sectional curvature. Let $\left(\Sigma_{2}, \theta_{2}, \beta_{2}\right)$ be a bound marked surface with holonomy in $\pi_{1}(M, Q)$ of genus 1 having 2 hyperbolic boundary components and a non-elementary marked handle $(a, b)$.

There exist bound marked pants $\left(T_{i}, \theta_{i}, \beta_{i}\right)_{1 \leqslant i \leqslant 2}$ with holonomy in $\pi_{1}(M)$ such that:

(1) $\left(\Sigma_{2}, \theta_{2}, \beta_{2}\right) \cong\left[\bigcirc\left(T_{1}, \theta_{1}, \beta_{1}\right)\right] \cup\left(T_{2}, \theta_{2}, \beta_{2}\right)$, and

(2) the images of $\theta_{1}$ and $\theta_{2}$ are Schottky groups.

We begin by proving the following proposition: 
Proposition 3.6.2 Let $(M, Q)$ be a pointed compact three dimensional manifold of strictly negative sectional curvature. Let $\left(\Sigma_{2}, \theta_{2}, \beta_{2}\right)$ be a bound marked surface with holonomy in $\pi_{1}(M, Q)$ of genus 1 having 2 boundary components and a non-elementary marked handle $\left(a_{1}, b_{1}\right)$.

Let $x_{1}$ and $y_{1}$ be the two oriented boundary components of $\Sigma_{2}$, and let $c$ be a curve in $\Sigma_{2}$ joining the base point of $y$ to that of $x$, but otherwise disjoint from $a_{1}, b_{1}, x_{1}$ and $y_{1}$. There exists a homeomorphism $\Psi$ of $\Sigma_{2}$ such that $\Psi_{*}\left(c^{-1} x_{1} c, y_{1}\right)$ and $\Psi_{*}\left(a_{1}^{-1} b_{1} a_{1}, b_{1}\right)$ are Schottky.

Proof Let $Q_{0}$ be the common base point of $a_{1}$ and $b_{1}$. Let $Q_{x}$ and $Q_{y}$ be the base points of $x$ and $y$ respectively. Let $\left(c_{0}, c_{x}, c_{y}\right)$ be a sash of $\left(Q_{0}, Q_{x}, Q_{y}\right)$. We denote:

$$
\begin{array}{ll}
a=c_{a}^{-1} a_{1} c_{a}, & b=c_{a}^{-1} b_{1} c_{a}, \\
x=c_{x}^{-1} x_{1} c_{x}, & y=c_{y}^{-1} y_{1} c_{y} .
\end{array}
$$

We may assume that $c=a^{-1} b^{-1} a b$ is freely homotopic to $(y x)^{-1}$. We denote:

$$
\alpha=\theta_{2}(a), \quad \beta=\theta_{2}(b), \quad \xi=\theta_{2}(x), \quad \eta=\theta_{2}(y), \quad \sigma=\theta_{2}(c) .
$$

By Proposition 3.5.2, we may suppose that $\langle\xi, \eta\rangle$ is a Schottky group and that $\langle\alpha, \beta\rangle$ is non-elementary. Let $T_{c}$ be the Dehn twist about $c$ such that, for all $m$ :

$$
\begin{array}{lll}
T_{c}^{m} a=a, & T_{c}^{m} b=b, \\
T_{c}^{m} x=c^{m} x c^{-m}, & T_{c}^{m} y=c^{m} y c^{-m} .
\end{array}
$$

By Proposition 2.1.4, after replacing $\theta$ with $\theta \circ\left(T_{c}\right)_{*}$ if necessary, we may suppose that $J=\xi \beta$ is not equal to the identity. For $k \in \mathbb{N}$, we define $d_{k}=x b a^{k}$. We may assume that $x b$ is freely homotopic to a simple closed curve, and, as in the proofs of Lemmata 3.4.1 and 3.5.1, using Dehn twists, for all $k$ and for all $n$, we may construct a homeomorphism $\Psi_{k, n}$ of $\Sigma_{2}$ such that:

$$
\begin{array}{ll}
\left(\Psi_{k, n}\right)_{*} a=d_{k}^{n} a, \quad\left(\Psi_{k, n}\right)_{*} b a^{k}=b a^{k}, \\
\left(\Psi_{k, n}\right)_{*} x=x, \quad\left(\Psi_{k, n}\right)_{*} y=d_{k}^{-n} y d_{k}^{n} .
\end{array}
$$

Where $d_{k}=x b a^{k}$. Choosing $k$ and $n$ as in Lemma 2.1.5, we obtain the desired result.

We now obtain Proposition 3.6.1:

Proof of Proposition 3.6.1 This follows directly by applying Proposition 3.6.2 and then cutting along curves freely homotopic to $y x$ and to $b$ as in the proof of Proposition 3.4.1.

\section{Invariant domains of Schottky groups}

\subsection{Introduction}

Throughout this section, $(M, Q)$ will be a pointed three dimensional Hadamard manifold and $\Gamma=\langle\alpha, \beta\rangle$ will be a $\operatorname{Schottky~subgroup~of~} \operatorname{Isom}(M, Q)$. In this section, we study the algebraic properties of Schottky groups.

We define an invariant domain of $\Gamma$ to be a Jordan domain $\Omega$ contained in $\partial_{\infty} M$ which is invariant under the action of $\Gamma$. Invariant domains are easy to construct. Indeed, let $\Gamma^{\prime}$ be any Schottky subgroup of $\mathbb{P} S L(2, \mathbb{C})$ which preserves the real line. Let $\phi: \Gamma \rightarrow \Gamma^{\prime}$ be an isomorphism, and let $\Phi: \partial_{\infty} M \rightarrow \hat{\mathbb{C}}$ be a homeomorphism which intertwines with $\phi$ 
(which we construct in Appendix A). $\Phi^{-1}(\hat{\mathbb{R}})$ is a Jordan curve in $\partial_{\infty} M$, and both connected components of its complement are invariant domains.

Trivially, for any invariant domain, $\Omega$ :

$$
\operatorname{Fix}(\Gamma) \subseteq \partial \Omega .
$$

We define $\hat{\Omega}$ by:

$$
\hat{\Omega}=\bar{\Omega} \backslash \operatorname{Fix}(\Gamma) .
$$

For every element $\gamma$ of $\Gamma$ we define $\gamma_{-}$and $\gamma_{+}$to be the repulsive and attractive fixed points of $\gamma$ respectively. We say that $\gamma$ is simple when there exists no other element $\eta$ in $\Gamma$ and no $n \geqslant 2$ such that $\gamma=\eta^{n}$. For $\gamma$ a simple element, we say that a connected component $I$ of $\partial \Omega \backslash \operatorname{Fix}(\Gamma)$ is adapted to $\gamma$ if and only if, viewed as an oriented subarc of $\partial \Omega$, it runs from $\gamma_{-}$to $\gamma_{+}$(and thus has $\gamma_{ \pm}$as its endpoints).

Such a connected component, when it exists, is unique. We say that $\Omega$ is adapted to the generators $(\alpha, \beta)$ when both of $\alpha$ and $\beta$ have adapted components in $\partial \Omega$. Trivially, the invariant domain $\Omega$, constructed as above is adapted to the generators $(\alpha, \beta)$.

Adaptation allows us to identify elements of the Schottky group with boundary components of the corresponding pant. Indeed, we show (Proposition 4.2.1) that $\Gamma$ acts properly discontinuously over $\hat{\Omega}$ and that its quotient is a compact topological surface with boundary. When $\Omega$ is adapted to the generators $(\alpha, \beta)$, the adapted components of $\alpha$ and $\beta$ project down to boundary components of $\hat{\Omega} / \Gamma$, and since $\alpha$ and $\beta$ cannot be conjugate in $\Gamma$, these components are distinct. Thus, since its fundamental group is the free group on two generators, $\hat{\Omega} / \Gamma$ is a pant.

This would already allow us to construct equivariant Plateau problems over a given bound, marked pant $(T, \theta, \beta)$ with holonomy in $\pi_{1}(M, Q)$ such that $\theta$ is a Schottky group. The solution is by no means unique, and in order to prove Theorem 1.0.4, we are required to study in more depth the algebraic properties of adapted invariant domains.

If $\gamma$ is a hyperbolic element of $\Gamma$, we define the torus $\mathbb{T}_{\gamma}$ by:

$$
\mathbb{T}_{\gamma}=\left(\partial_{\infty} \tilde{M} \backslash\left\{\gamma_{ \pm}\right\}\right) /\langle\gamma\rangle .
$$

For any hyperbolic element $\gamma$ of $\Gamma$, we canonically define a preferred one dimensional subspace $L_{\gamma}$ of $H_{1}\left(\mathbb{T}_{\gamma}\right)$. We may also associate to any invariant domain $\Omega$ of $\Gamma$ a unique element $[\Omega]_{\gamma}$ in $H_{1}\left(\mathbb{T}_{\gamma}\right)$. This element necessarily lies in $L_{\gamma}$ (see Sect. 4.4).

Let $\mathrm{Homeo}_{0}\left(\partial_{\infty} M\right)$ denote the connected component of the space of homeomorphisms of $\partial_{\infty} M$ which contains the identity. There exists a canonical homomorphism of $\pi_{1}(M, Q)$ into $\mathrm{Homeo}_{0}\left(\partial_{\infty} M\right)$. We may show (see Corollary 4.3.2) that if $\widetilde{\mathrm{Homeo}}_{0}\left(\partial_{\infty} M\right)$ denotes the universal cover of $\mathrm{Homeo}_{0}\left(\partial_{\infty} M\right)$, then it is a two-fold covering. For every hyperbolic element $\gamma$ of $\Gamma$, we may canonically define a mapping $\operatorname{Lift}_{\gamma}: L_{\gamma} \rightarrow \mathrm{Homeo}_{0}\left(\partial_{\infty} M\right)$.

The main result of this section, which allows us to establish the obstruction to constructing a $\pi_{1}(M, Q)$ structure over a closed surface $\Sigma$ with holonomy in $\pi_{1}(M, Q)$ may now be expressed as follows:

Lemma 4.1.1 Let $\Gamma=\langle\alpha, \beta\rangle$ be a Schottky group. Let us denote $\gamma=\alpha \beta$. Let $\left([a]_{\alpha},[b]_{\beta}\right.$, $[c]_{\gamma}$ ) be a triplet in $L_{\alpha} \times L_{\beta} \times L_{\gamma}$. There exists an invariant domain, $\Omega$, of $\Gamma$ in $\partial_{\infty} M$ adapted to the generators $(\alpha, \beta)$ such that:

$$
[\Omega]_{\alpha}=[a]_{\alpha}, \quad[\Omega]_{\beta}=[b]_{\beta}, \quad[\Omega]_{\gamma}=[c]_{\gamma},
$$

if and only if:

$$
\operatorname{Lift}_{\gamma}\left([c]_{\gamma}\right)^{-1} \operatorname{Lift}_{\alpha}\left([a]_{\alpha}\right) \operatorname{Lift}_{\beta}\left([b]_{\beta}\right) \neq \operatorname{Id} .
$$


The proof of this result follows immediately from Propositions 4.5.2 and 4.5.3.

In the second part of this section, we review the geometric properties of invariant domains, justifying the assertions made in this introduction. In the third part, we review the topology of the group of homeomorphisms of the sphere and the properties of braid groups of order three in the sphere. In the fourth part, we define the element $[\Omega]_{\gamma}$, for any hyperbolic element, $\gamma$, of $\Gamma$ and any invariant domain, $\Omega$, of $\Gamma$. Finally, in the fifth part, we prove Propositions 4.5.2 and 4.5.3.

\subsection{Invariant domains}

In this section, we study the geometric properties of invariant domains. The following result is fairly trivial:

Proposition 4.2.1 $\Gamma$ acts properly discontinuously on $\hat{\Omega}$ and $\hat{\Omega} / \Gamma$ is compact.

Since the fundamental group of $\hat{\Omega} / \Gamma$ is isomorphic to the free group on two generators, $\hat{\Omega} / \Gamma$ is either a pant or a punctured torus. We consequently restrict our attention to invariant domains which are adapted to these generators, since, in this case, the adapted components of $\alpha$ and $\beta$ project down to distinct boundary components of $\hat{\Omega} / \Gamma$ and $\hat{\Omega} / \Gamma$ is consequently a pant.

In the sequel, we will require the following technical result concerning adapted invariant domains:

Proposition 4.2.2 Let $\Omega$ be an invariant domain of $\Gamma$ adapted to the generators $(\alpha, \beta)$. Let $\gamma$ and $\delta$ be the elements of $\Gamma$ defined by:

$$
\gamma=\alpha \beta, \quad \delta=\beta \alpha .
$$

If $\alpha_{ \pm}, \beta_{ \pm}, \gamma_{ \pm}$and $\delta_{ \pm}$are the fixed points of these four elements, then they are distributed round $\partial \Omega$ in the following order:

$$
\alpha_{-}, \alpha_{+}, \delta_{+}, \delta_{-}, \beta_{-}, \beta_{+}, \gamma_{+}, \gamma_{-} .
$$

Proof Let $p$ be one of the fixed points of $\gamma$. We have:

$$
(p \cdot \alpha) \cdot \delta=p \cdot(\alpha \beta \alpha)=(p \cdot \gamma) \cdot \alpha=p \cdot \alpha .
$$

Consequently, $\alpha$ sends the fixed points of $\gamma$ to those of $\delta$, and so $\gamma_{ \pm} \cdot \alpha=\delta_{ \pm}$. Likewise $\delta_{ \pm} \cdot \beta=\gamma_{ \pm}$. Let $I_{\alpha}$ and $I_{\beta}$ be the adapted components of $\alpha$ and $\beta$ respectively.

Since $\alpha$ preserves orientation, it also preserves $I_{\alpha}$ and $I_{\alpha}^{C}$, Moreover, it shifts all points in $I_{\alpha}$ and $I_{\alpha}^{C}$ towards $\alpha^{+}$. Consequently, the attractive and repulsive fixed points of $\alpha, \gamma$ and $\delta$ appear in the following order as one moves around $\partial \Omega$ in the positive direction:

$$
\alpha_{-}, \alpha_{+}, \delta_{ \pm}, \gamma_{ \pm}
$$

Similarly, the mapping $\beta$ shifts all points in $I_{\beta}$ and $I_{\beta}^{C}$ towards $\beta^{+}$. Thus, the attractive and repulsive fixed points of $\beta, \gamma$ and $\delta$ appear in the following order as one moves around $\partial \Omega$ in the positive direction:

$$
\beta_{-}, \beta_{+}, \gamma_{ \pm}, \delta_{ \pm}
$$

Since the mapping $\alpha$, which sends the fixed points of $\gamma$ to those of $\delta$, is an orientation preserving mapping, it follows that, as one moves round $\partial \Omega$ in the positive direction, $\gamma_{-}$and 
$\gamma_{+}$appear in the same order as $\delta_{-}$and $\delta_{+}$. Moreover, since $I_{\alpha}$ and $I_{\beta}$ are adapted components, they have no fixed points of $\Gamma$ in their interiors, and so, combining all this information, we find that these eight fixed points appear round $\partial \Omega$ in the following order:

$$
\alpha_{-}, \alpha_{+}, \delta_{ \pm}, \delta_{\mp}, \beta_{-}, \beta_{+}, \gamma_{ \pm}, \gamma_{\mp} \text {. }
$$

Let $p$ be the fixed point of $\gamma$ lying closest to $\beta_{+}$in $I_{\beta}^{C}$. Let $q$ be the fixed point of $\delta$ lying closest to $\alpha_{+}$in $I_{\alpha}^{C}$. The point $p$ is also the fixed point of $\gamma$ lying closest to $\alpha_{+}$in $I_{\alpha}^{C}$. Since $\alpha$ preserves orientation, we have $p \cdot \alpha=q$. The points $\alpha_{ \pm}$lie between $q$ and $\beta_{+}$in $I_{\beta}^{C}$. Consequently, the points $\alpha_{ \pm} \cdot \gamma=\alpha_{ \pm} \cdot \beta$ lie between $p=p \cdot \gamma=q \cdot \beta$ and $\beta_{+}$in $I_{\beta}^{C}$.

Let $I_{1}$ and $I_{2}$ be the two connected components of $\partial \Omega \backslash\left\{\gamma_{ \pm}\right\}$. The mapping $\gamma$ shifts all points in the intervals $I_{1}$ and $I_{2}$ towards $\gamma_{+}$. We may suppose that $I_{1}$ is the component containing $\alpha_{ \pm}$. Since $\alpha_{+} \cdot \gamma$ lies between $p$ and $\beta_{+}$in $I_{\beta}^{C}$, it follows that it also lies between $\alpha_{+}$ and $p$ in $I_{1}$. The point $p$ is consequently the attractive fixed point of $\gamma$ and the result now follows.

\subsection{Braids and the topology of $\operatorname{Homeo}_{0}\left(\partial_{\infty} M\right)$}

For any topological surface $\Sigma$, let $\operatorname{Homeo}_{0}(\Sigma)$ be the connected component of $\operatorname{Homeo}(\Sigma)$ that contains the identity. There exists a canonical embedding of the group $\pi_{1}(M, Q)$ into Homeo $_{0}\left(\partial_{\infty} M\right)$. We may thus consider the mapping $\theta$ as a homomorphism of $\pi_{1}\left(\Sigma, P_{0}\right)$ taking values in the group $\operatorname{Homeo}_{0}\left(\partial_{\infty} M\right)$.

Let $X \subseteq Y$ be topological spaces. We define a strong deformation retraction of $Y$ onto $X$ to be a mapping $\psi: I \times Y \rightarrow Y$ such that:

(i) $\psi_{0}: Y \rightarrow Y$ is the identity,

(ii) $\psi_{1}(Y) \subseteq X$, and

(iii) for all $t \in I$, the restriction of $\psi_{t}$ to $X$ is the identity.

Let us denote by $S^{2} \subseteq \mathbb{R}^{3}$ the sphere of radius 1 in $\mathbb{R}^{3}$. We recall the following result concerning the homotopy type of $\operatorname{Homeo}_{0}\left(S^{2}\right)$ (see $\left.[3,6]\right)$ :

Theorem 4.3.1 (Friberg [3]) The space $\mathrm{Homeo}_{0}\left(S^{2}\right)$ strong deformation retracts onto $\mathrm{SO}(3, \mathbb{R})$.

In particular, we obtain:

\section{Corollary 4.3.2}

$$
\pi_{1}\left(\operatorname{Homeo}_{0}\left(\partial_{\infty} M\right), \mathrm{Id}\right) \cong \pi_{1}(\mathrm{SO}(3, \mathbb{R}), \mathrm{Id}) \cong \mathbb{Z}_{2} .
$$

A braid of order 3 in $\partial_{\infty} M$ is a triple $\gamma=\left(\gamma_{1}, \gamma_{2}, \gamma_{3}\right)$ where $\gamma_{1}, \gamma_{2}, \gamma_{3}: I \rightarrow S^{2}$ are curves such that, for all $t \in I$, the points $\gamma_{1}(t), \gamma_{2}(t)$ and $\gamma_{3}(t)$ are all distinct. The interested reader may find a more detailed treatment of braids in general in [9] or (equivalently) in Appendix D of [7]. For all $t \in I$, we denote:

$$
\gamma(t)=\left(\gamma_{1}(t), \gamma_{2}(t), \gamma_{3}(t)\right)
$$

We call the point $\gamma(0)$ the base point of the braid $\gamma$. We say that the braid is closed if and only if $\boldsymbol{\gamma}(0)=\boldsymbol{\gamma}(1)$. Let $\boldsymbol{\gamma}_{0}$ and $\boldsymbol{\gamma}_{1}$ be two braids having the same endpoints. A homotopy between $\boldsymbol{\gamma}_{0}$ and $\boldsymbol{\gamma}_{1}$ is a continuous family $\left(\boldsymbol{\eta}_{t}\right)_{t \in I}$ of braids having the same extremities as $\boldsymbol{\gamma}_{0}$ and $\boldsymbol{\gamma}_{1}$ such that:

$$
\eta_{0}=\gamma_{0}, \quad \eta_{1}=\gamma_{1}
$$


For $\mathbf{p}=\left(p_{1}, p_{2}, p_{3}\right)$ a triplet of distinct points in $\partial_{\infty} M$, we denote by $T_{\mathbf{p}}$ the family of braids of order 3 in $\partial_{\infty} M$ having $\mathbf{p}$ as a base point. Likewise, we denote by $T_{\mathbf{p}}^{0}$ the subfamily of $T_{\mathbf{p}}$ consisting of all the closed braids in $T_{\mathbf{p}}$. Let $\sim$ be the homotopy equivalence relation over $T_{\mathbf{p}}$. The law of composition of curves yields the law of composition of braids, and the set $T_{\mathbf{p}} / \sim$ thus forms a semigroup. Likewise, $T_{\mathbf{p}}^{0} / \sim$ forms a group. For $\boldsymbol{\gamma}=\left(\gamma_{1}, \gamma_{2}, \gamma_{3}\right)$ a braid in $T_{\mathbf{p}}$, we denote by $[\boldsymbol{\gamma}]=\left[\gamma_{1}, \gamma_{2}, \gamma_{3}\right]$ its projection in $T_{\mathbf{p}} / \sim$.

Let $C_{0}\left(I, \mathrm{Homeo}_{0}\left(\partial_{\infty} M\right)\right)$ denote the family of continuous curves in $\mathrm{Homeo}_{0}\left(\partial_{\infty} M\right)$ leaving from the identity. For any triplet $\mathbf{p}=\left(p_{1}, p_{2}, p_{3}\right)$ of distinct points in $\partial_{\infty} M$, we define the mapping $\mathcal{T}_{\mathbf{p}}$ from $C_{0}\left(I, \mathrm{Homeo}_{0}\left(\partial_{\infty} M\right)\right)$ to $T_{\mathbf{p}}$ such that, for all $c \in C_{0}\left(I, \mathrm{Homeo}_{0}\left(\partial_{\infty}\right.\right.$ $M)$ ), and for all $t \in I$ :

$$
\left(\mathcal{T}_{\mathbf{p}} c\right)(t)=\left(p_{1} \cdot c(t), p_{2} \cdot c(t), p_{3} \cdot c(t)\right) .
$$

In the case where $\partial_{\infty} M=\hat{\mathbb{C}}$, for all $\mathbf{p}$, the mapping $\mathcal{T}_{\mathbf{p}}$ restricts to a homeomorphism from $C_{0}(I, \mathbb{P} S L(2, \mathbb{C}))$ onto $T_{\mathbf{p}}$. Consequently, in the general case, the mapping $\mathcal{T}_{\mathbf{p}}$ is surjective. Moreover, for all $\mathbf{p}$, the mapping $\mathcal{T}_{\mathbf{p}}$ quotients down to a surjective mapping from $\widetilde{\mathrm{Homeo}}_{0}\left(\partial_{\infty} M\right)$ to $T_{\mathbf{p}} / \sim$ which we also denote by $\mathcal{T}_{\mathbf{p}}$. By Theorem 4.3.1, this mapping is bijective.

Let $\gamma$ be a braid in $T_{\mathbf{p}}$. We may suppose that there exists a point $p_{\infty}$ in $\partial_{\infty} M$ which does not lie in the image of $\gamma$. Let $\alpha: \partial_{\infty} M \backslash\left\{p_{\infty}\right\} \rightarrow \mathbb{R}^{2}$ be a homeomorphism. For any closed curve $\eta$ in $\mathbb{R}^{2} \backslash\{0\}$, let Wind $(\eta)$ be the winding number of $\eta$ about 0 . The quantity:

$$
\sum_{i<j} \operatorname{Wind}\left(\alpha \circ \gamma_{i}-\alpha \circ \gamma_{j}\right) \operatorname{Mod} 2
$$

is well defined and independent of $\alpha$ and $p_{\infty}$ (see, for example, [9], or appendix $D$ of [7]). We thus define $\operatorname{Wind}_{r}(\boldsymbol{\gamma})$, the relative winding number of $\boldsymbol{\gamma}$ to be equal to this quantity.

Wind $_{r}$ defines an isomorphism from $T_{\mathbf{p}}^{0} / \sim$ to $\mathbb{Z}_{2}$. We define the mapping $W_{r, \boldsymbol{p}}$ which sends $\pi_{1}\left(\operatorname{Homeo}_{0}\left(\partial_{\infty} M\right)\right)$ into $\mathbb{Z}_{2}$ by:

$$
W_{r, \boldsymbol{p}}=\text { Wind }_{r} \circ \mathcal{T}_{\mathbf{p}} .
$$

The mapping $W_{r, \boldsymbol{p}}$ defines an isomorphism from $\pi_{1}\left(\operatorname{Homeo}_{0}\left(\partial_{\infty} M\right)\right)$ to $\mathbb{Z}_{2}$. Moreover, it does not depend on the choice of $\mathbf{p}$, and we thus denote $W_{r}=W_{r, \boldsymbol{p}}$.

\subsection{Homological classes}

For $\gamma \in \Gamma$, we define the torus $\mathbb{T}_{\gamma}$ by:

$$
\mathbb{T}_{\gamma}=\left(\partial_{\infty} M \backslash\left\{\gamma_{-}, \gamma_{+}\right\}\right) /\langle\gamma\rangle \text {. }
$$

Let $C$ be a closed curve which turns once around the cylinder $\partial_{\infty} M \backslash\left\{\gamma_{ \pm}\right\}$in such a manner that $\gamma_{+}$lies to its left (in other words, in its interior). $C$ defines a unique homology class $[C]_{\gamma}$ in $H_{1}\left(\mathbb{T}_{\gamma}\right)$. Using Poincare duality, we define $L_{\gamma} \subseteq H_{1}\left(\mathbb{T}_{\gamma}\right)$ by:

$$
L_{\gamma}=\left\{[a] \in H_{1}\left(\mathbb{T}_{\gamma}\right) \mid\left\langle\left[C_{\gamma}\right],[a]\right\rangle=1\right\} .
$$

Heuristically, $L_{\gamma}$ is a straight line which contains all the curves in $\mathbb{T}_{\gamma}$ which cross $C_{\gamma}$ exactly once, going from right to left. For $[a],[b] \in L_{\gamma}$, we define $[a]-[b]$ to be the unique integer $n$ such that:

$$
[a]=[b]+n[C]_{\gamma} .
$$

Conversely, for $[a] \in L_{\gamma}$ and $n \in \mathbb{Z}$, we define $[a]+n \in L_{\gamma}$ such that $([a]+n)-[a]=n$. 
Let us denote by $\pi_{\gamma}$ the canonical projection from $\partial_{\infty} M \backslash\left\{\gamma_{-}, \gamma_{+}\right\}$onto $\mathbb{T}_{\gamma}$. Let $\Omega$ be an invariant domain of $\Gamma$ in $\partial_{\infty} M$. If $c: I \rightarrow \Omega$ is a curve such that $c(0)=\gamma_{-}$and $c(1)=\gamma_{+}$, then the homology class of $\pi_{\gamma} \circ c$ in $\mathbb{T}_{\gamma}$ lies in $L_{\gamma}$. Moreover, it does not depend on $c \subseteq \Omega$, and we thus define:

$$
[\Omega]_{\gamma}=\left[\pi_{\gamma} \circ c\right] \in L_{\gamma}
$$

\subsection{Liftings of mappings}

Let $\gamma$ be an element in $\Gamma$. Let $\gamma_{ \pm}$be the fixed points of $\gamma$. Let $c: I \rightarrow \partial_{\infty} M \backslash\left\{\gamma_{ \pm}\right\}$be a curve such that:

$$
c(0) \cdot \gamma=c(1)
$$

The curve $c$ projects down to an element of $L_{\gamma}$. We consider $\gamma_{ \pm}$as being constant curves, and we define $\hat{\gamma}_{c}$ to be the unique lifting of $\gamma$ in $\widetilde{\mathrm{Homeo}}_{0}\left(\partial_{\infty} M\right)$ such that:

$$
\mathcal{T}_{\left(\gamma_{-}, c(0), \gamma_{+}\right)} \hat{\gamma}_{c}=\left[\gamma_{-}, c, \gamma_{+}\right] \text {. }
$$

Trivially, $\hat{\gamma}_{c}$ only depends on the homotopy class of $\left[\pi_{\gamma} \circ c\right]$ in $L_{\gamma} \subseteq H_{1}\left(\mathbb{T}_{\gamma}\right)$. We thus obtain a mapping Lift $\gamma: L_{\gamma} \rightarrow \mathrm{Homeo}_{0}\left(\partial_{\infty} M\right)$ which is defined such that, for all $c$ with $c(0) \cdot \gamma=c(1)$ :

$$
\operatorname{Lift}_{\gamma}\left(\left[\pi_{\gamma} \circ c\right]\right)=\hat{\gamma}_{c}
$$

Proposition 4.5.1 Let $c, c^{\prime}: I \rightarrow M \backslash\left\{\gamma_{ \pm}\right\}$be curves such that $c(0) \cdot \gamma=c(1)$ and $c^{\prime}(0) \cdot \gamma=c^{\prime}(1)$. Then:

$$
\operatorname{Lift}_{\gamma}\left(\left[\pi_{\gamma} \circ c\right]\right)=\operatorname{Lift}_{\gamma}\left(\left[\pi_{\gamma} \circ c^{\prime}\right]\right) \Leftrightarrow\left[\pi_{\gamma} \circ c\right]-\left[\pi_{\gamma} \circ c^{\prime}\right]=0 \operatorname{Mod} 2 .
$$

Proof Let us define:

$$
\alpha=\operatorname{Lift}_{\gamma}\left(\left[\pi_{\gamma} \circ c\right]\right)^{-1} \cdot \operatorname{Lift}_{\gamma}\left(\left[\pi_{\gamma} \circ c^{\prime}\right]\right) .
$$

The element $\alpha$ is a lifting of the identity in $\widetilde{H o m e o}_{0}\left(\partial_{\infty} M\right)$, and:

$$
\mathcal{T}_{\left(\gamma_{-}, c^{\prime}(0), \gamma_{+}\right)} \alpha=\left[\gamma_{-}, c^{-1} c^{\prime}, \gamma_{+}\right]
$$

The element $\alpha$ is equal to the identity if and only if:

$$
W_{r}\left[\gamma_{-}, c^{-1} c^{\prime}, \gamma_{+}\right]=0 .
$$

However, since $\gamma_{ \pm}$are trivial paths, we obtain:

$$
W_{r}\left[\gamma_{-}, c^{-1} c^{\prime}, \gamma_{+}\right]=\left[\pi_{\gamma} \circ c^{\prime}\right]-\left[\pi_{\gamma} \circ c\right] \operatorname{Mod} 2 .
$$

The result now follows.

If $\Omega$ is an invariant domain of $\Gamma$ in $\partial_{\infty} M$, then, for every element $\gamma \in \Gamma$, we define $\hat{\gamma}_{\Omega}$ by:

$$
\hat{\gamma}_{\Omega}=\operatorname{Lift}_{\gamma}[\Omega]_{\gamma}
$$

We obtain the following result: 


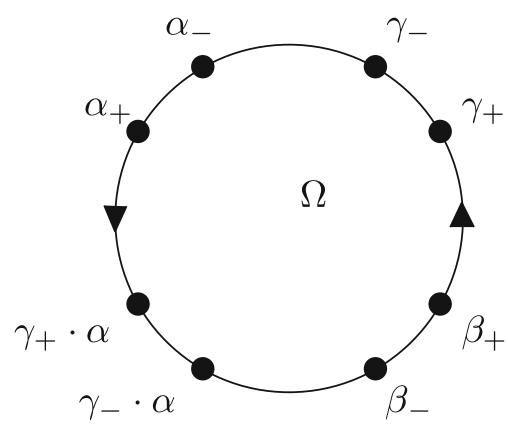

Fig. 2

Proposition 4.5.2 Let $\Gamma=\langle\alpha, \beta\rangle$ be a Schottky group. Let $\Omega$ be an invariant domain of $\Gamma$ in $\partial_{\infty} M$ which is adapted to the generators $(a, b)$. Let us denote $\gamma=\alpha \beta$, and let us define $\Delta$ by:

$$
\Delta=\hat{\gamma}_{\Omega}^{-1} \hat{\alpha}_{\Omega} \hat{\beta}_{\Omega}
$$

Then $\Delta$, which is a closed curve in $\mathrm{Homeo}_{0}\left(\partial_{\infty} M\right)$, is homotopically non-trivial.

Proof By Lemma A.1.2, it suffices to prove this result when $\partial_{\infty} \tilde{M}=\hat{\mathbb{C}}$ and $\Gamma$ is a subgroup of $\mathbb{P} S L(2, \mathbb{C})$. In this case, for any triplet $\mathbf{p}=\left(p_{1}, p_{2}, p_{3}\right)$ of distinct points in $\hat{\mathbb{C}}$, the restriction of the mapping $\mathcal{T}_{\mathbf{p}}$ to $C_{0}(I, \mathbb{P} S L(2, \mathbb{C}))$ is bijective. Let $\mathcal{S}_{\mathbf{p}}$ denote its inverse. Since, for any braid, $\boldsymbol{\eta}$ in $T_{\mathbf{p}}$, the point $\mathbf{p}$ is the base point of $\boldsymbol{\eta}$, and is thus determined by $\boldsymbol{\eta}$, we may write $\mathcal{S}$ instead of $\mathcal{S}_{\mathbf{p}}$.

$\partial \Omega$ is homeomorphic to the circle $S^{1}$ and is invariant under the action of $\Gamma$ on $\mathbb{C}$. By Proposition 4.2.2, since $\Omega$ is adapted to the generators $(\alpha, \beta)$, the 8 points $\alpha_{ \pm}, \beta_{ \pm}, \gamma_{ \pm}$and $\gamma_{ \pm} \cdot \alpha$ are distributed around $\partial \Omega$ according to the diagram in Fig. 2.

For every point, $p$ in $\partial_{\infty} M$, let us denote also by $p$ the constant curve which sends the unit interval onto $p$. Let $p_{0}$ be a point in $\Omega$. Let $a$ be a curve in $\Omega$ joining $p_{0}$ to $p_{0} \cdot \alpha$. Let $b$ be a curve in $\Omega$ joining $p_{0} \cdot \alpha$ to $p_{0} \cdot(\alpha \beta)$. By definition:

$$
\begin{aligned}
\hat{\alpha}_{\Omega} & =\left[\mathcal{S}\left(\alpha_{-}, a, \alpha_{+}\right)\right], \\
\hat{\beta}_{\Omega} & =\left[\mathcal{S}\left(\beta_{-}, b, \beta_{+}\right)\right], \\
\hat{\gamma}_{\Omega}^{-1} & =\left[\mathcal{S}\left(\gamma_{-},(a \cdot b)^{-1}, \gamma_{+}\right)\right] .
\end{aligned}
$$

Let $\xi_{ \pm}: I \rightarrow \partial \Omega$ be such that $\xi_{ \pm}$avoids $\alpha_{\mp}$ and that:

$$
\xi_{ \pm}(0)=\alpha_{ \pm}, \quad \xi_{ \pm}(1)=\gamma_{ \pm} .
$$

Since $\partial \Omega$ is invariant under the action of $\alpha$, the curves $\xi_{ \pm} \cdot \alpha$ lie in $\partial \Omega$ and join $\alpha_{ \pm}$to $\gamma_{ \pm} \cdot \alpha$ whilst avoiding $\alpha_{\mp}$. Let $\left(\aleph_{t}^{ \pm}\right)_{t \in I}$ be a continuous family of curves in $\partial \Omega$ such that:

(i) for all $s$ :

$$
\aleph_{0}^{ \pm}(s)=\alpha_{ \pm},
$$

and,

(ii) for all $t$ :

$$
\begin{aligned}
& \aleph_{t}^{ \pm}(0)=\xi_{ \pm}(t), \\
& \aleph_{t}^{ \pm}(1)=\xi_{ \pm}(t) \cdot \alpha .
\end{aligned}
$$


To be more precise, we deform $\aleph^{-}$slightly towards the interior of $\partial \Omega$ and $\aleph^{+}$slightly towards the exterior of $\partial \Omega$ so that they do not intersect each other. Heuristically, $\aleph_{1}^{ \pm}$is a curve which goes from $\gamma_{ \pm}$to $\gamma_{ \pm} \cdot \alpha$ in the clockwise direction. For all $t$ and for all $s$, we may suppose that the three points $\left(\aleph_{t}^{-}(0), a(0), \aleph_{t}^{+}(0)\right)$ are distinct. Moreover, for all $t$ :

$$
\left(\aleph_{t}^{-}(0), a(0), \aleph_{t}^{+}(0)\right) \cdot \alpha=\left(\aleph_{t}^{-}(1), a(1), \aleph_{t}^{+}(1)\right) .
$$

Consequently, the family $\left(\aleph_{t}^{-}, a, \aleph_{t}^{+}\right)_{t \in I}$ defines a homotopy between the braids $\left(\alpha_{-}, a\right.$, $\left.\alpha_{+}\right)$and $\left(\aleph_{1}^{-}, a, \aleph_{1}^{+}\right)$, respecting the fact that $\alpha$ sends the start point of each braid onto its end point. Since the set of liftings of $\alpha$ in $\widetilde{\mathbb{P S L}}(2, \mathbb{C})$ is discrete, it follows by continuity that:

$$
\left[\mathcal{S}\left(\aleph_{1}^{-}, a, \aleph_{1}^{+}\right)\right]=\left[\mathcal{S}\left(\alpha_{-}, a, \alpha_{+}\right)\right] .
$$

We define $\left(\beth_{t}^{ \pm}\right)_{t \in I}$ in a similar fashion using $\beta$ instead of $\alpha$, and we obtain:

$$
\begin{aligned}
\hat{\gamma}_{T}^{-1} \hat{\alpha}_{T} \hat{\beta}_{T} & =\left[\mathcal{S}\left(\gamma_{-},(a \cdot b)^{-1}, \gamma_{+}\right) \mathcal{S}\left(\alpha_{-}, a, \alpha_{+}\right) \mathcal{S}\left(\beta_{-}, b, \beta_{+}\right)\right] \\
& =\left[\mathcal{S}\left(\gamma_{-},(a \cdot b)^{-1}, \gamma_{+}\right) \mathcal{S}\left(\aleph_{1}^{-}, a, \aleph_{1}^{+}\right) \mathcal{S}\left(\beth_{1}^{-}, b, \beth_{1}^{+}\right)\right] \\
& =\left[\mathcal{S}\left(\gamma_{-} \cdot \aleph_{1}^{-} \cdot \beth_{1}^{-},(a \cdot b)^{-1} \cdot a \cdot b, \gamma_{+} \cdot \aleph_{1}^{+} \cdot \beth_{1}^{+}\right)\right] .
\end{aligned}
$$

We thus define the closed braid $\mathcal{T}$ by:

$$
\mathcal{T}=\left(\gamma_{-} \cdot \aleph_{1}^{-} \cdot \beth_{1}^{-},(a \cdot b)^{-1} \cdot a \cdot b, \gamma_{+} \cdot \aleph_{1}^{+} \cdot \beth_{1}^{+}\right) .
$$

Since $\aleph_{1}^{ \pm}$and $\beth_{1}^{ \pm}$stay close to $\partial \Omega$ and $(a \cdot b)^{-1} \cdot a \cdot b$ lies in the interior of $\Omega$, there exists a homotopy between $(a \cdot b)^{-1} \cdot a \cdot b$ and the constant curve $p_{0}$ which stays away from $\aleph_{1}^{ \pm}$ and $\beth_{1}^{ \pm}$. Consequently:

$$
\begin{aligned}
\mathcal{T} & \sim\left(\gamma_{-} \cdot \aleph_{1}^{-} \cdot \beth_{1}^{-}, p_{0}, \gamma_{+} \cdot \aleph_{1}^{+} \cdot \beth_{1}^{+}\right) \\
& \sim\left(\aleph_{1}^{-} \cdot \beth_{1}^{-}, p_{0}, \aleph_{1}^{+} \cdot \beth_{1}^{+}\right) .
\end{aligned}
$$

Heuristically, $\aleph_{1}^{+} \cdot \beth_{1}^{+}$is a curve which turns once about $\partial \Omega$ in the clockwise direction. Moreover, this curve lies to the exterior of $\partial \Omega$. There thus exists a homotopy between this curve and the constant curve $q_{+}$which stays away from $p_{0}$ and $\aleph_{1}^{-} \cdot \beth_{1}^{-}$. Thus:

$$
\mathcal{T} \sim\left(\aleph_{1}^{-} \cdot \beth_{1}^{-}, p_{0}, q_{+}\right) .
$$

Finally, heuristically, $\aleph_{1}^{-} \cdot \beth_{1}^{-}$is a simple closed curve which separates $p_{0}$ from $q_{+}$. Consequently:

$$
\begin{aligned}
& \operatorname{Wind}_{r}(\mathcal{T})=1 \\
& \Rightarrow W_{r}\left(\hat{\gamma}_{\Omega}^{-1} \hat{\alpha}_{\Omega} \hat{\beta}_{\Omega}\right)=1 .
\end{aligned}
$$

The result now follows.

We also obtain the converse to this result:

Proposition 4.5.3 Let $\Gamma=\langle\alpha, \beta\rangle$ be a Schottky group. If $[a]_{\alpha} \in L_{\alpha},[b]_{\beta} \in L_{\beta}$ and $[c]_{\gamma} \in L_{\gamma}$ are such that:

$$
\left(\operatorname{Lift}_{\gamma}[c]_{\gamma}\right)^{-1}\left(\operatorname{Lift}_{\alpha}[a]_{\alpha}\right)\left(\operatorname{Lift}_{\beta}[b]_{\beta}\right) \neq \mathrm{Id},
$$

then there exists an invariant domain $\Omega$ of $\partial_{\infty} M$ adapted to the generators $(\alpha, \beta)$ such that:

$$
[\Omega]_{\alpha}=[a]_{\alpha}, \quad[\Omega]_{\beta}=[b]_{\beta}, \quad[\Omega]_{\gamma}=[c]_{\gamma} .
$$


Proof By Lemma A.1.2, it suffices to prove this result in the case where $\partial_{\infty} M=\hat{\mathbb{C}}$ and $\Gamma$ is a Schottky subgroup of $\mathbb{P} S L(2, \mathbb{C})$.

Let $\left(C_{\alpha}^{ \pm}, C_{\beta}^{ \pm}\right)$be generating circles of $\Gamma$ with respect to the generators $(\alpha, \beta)$. We define the set $X \subseteq \hat{\mathbb{C}}$ by:

$$
X=\hat{\mathbb{C}} \backslash\left(\operatorname{Int}\left(C_{\alpha}^{+}\right) \cup \operatorname{Int}\left(C_{\alpha}^{-}\right) \cup \operatorname{Int}\left(C_{\beta}^{+}\right) \cup \operatorname{Int}\left(C_{\beta}^{-}\right)\right) .
$$

We now define the curves $\left(a, b, c_{1}, c_{2}\right)$ to be non-intersecting, simple curves, lying in the interior of $X$ except at their end points, such that:

$$
\begin{aligned}
& a(1)=a(0) \cdot \alpha, \quad b(1)=b(0) \cdot \beta, \\
& c_{1}(0)=c_{2}(1) \cdot \alpha, \quad c_{2}(0)=c_{2}(1) \cdot \beta .
\end{aligned}
$$

We refer to the quadruplet $\left(a, b, c_{1}, c_{2}\right)$ as generating curves for $\Gamma$ with respect to the generating circles $\left(C_{\alpha}^{ \pm}, C_{\beta}^{ \pm}\right)$. By taking the images of these four curves under the actions of elements of $\gamma$, and by then adjoining $\operatorname{Fix}(\gamma)$, we obtain uniquely from these four curves a Jordan curve which is invariant under the action of $\Gamma$ and which we denote by $\Gamma\left(a, b, c_{1}, c_{2}\right)$. The interior of $\Gamma\left(a, b, c_{1}, c_{2}\right)$ is an invariant domain of $\Gamma$ which is adapted to the generators $(\alpha, \beta)$. Let us denote this domain by $\Omega\left(a, b, c_{1}, c_{2}\right)$. By definition:

$$
[a]_{\alpha}=\left[\Omega\left(a, b, c_{1}, c_{2}\right)\right]_{\alpha}, \quad[b]_{\beta}=\left[\Omega\left(a, b, c_{1}, c_{2}\right)\right]_{\beta} .
$$

We define the curve $c$ by $c=c_{2}^{-1}\left(c_{1} \cdot \beta^{-1}\right)^{-1}$, and we observe that:

$$
c(1)=c(0) \cdot \gamma \text {. }
$$

Consequently:

$$
[c]_{\gamma}=\left[\Omega\left(a, b, c_{1}, c_{2}\right)\right]_{\gamma} .
$$

By Proposition 4.5.2:

$$
\operatorname{Lift}_{\gamma}\left([c]_{\gamma}\right)^{-1} \operatorname{Lift}_{\beta}\left([b]_{\beta}\right) \operatorname{Lift}_{\alpha}\left([a]_{\alpha}\right) \neq \mathrm{Id} .
$$

Let $T_{1}$ be the Dehn twist about $C_{\alpha}^{+}$such that:

$$
T_{1}[a]_{\alpha}=[a]_{\alpha}+1, \quad T_{1}[b]_{\beta}=[b]_{\beta}, \quad T_{1}[c]_{\gamma}=[c]_{\gamma}+1 .
$$

Let $T_{2}$ be the Dehn twist about $C_{\beta}^{+}$such that:

$$
T_{2}[a]_{\alpha}=[a]_{\alpha}, \quad T_{2}[b]_{\beta}=[b]_{\beta}+1, \quad T_{2}[c]_{\gamma}=[c]_{\gamma}+1 .
$$

Finally, let $T_{3}$ be a Dehn twist about a curve that separates $C_{\alpha}^{ \pm}$from $C_{\beta}^{ \pm}$. We may choose $T_{3}$ such that:

$$
T_{3}[a]_{\alpha}=[a]_{\alpha}, \quad T_{3}[b]_{\beta}=[b]_{\beta}, \quad T_{3}[c]_{\gamma}=[c]_{\gamma}+2 .
$$

Using combinations of these three Dehn twists, for any triple $(x, y, z) \in L_{\alpha} \times L_{\beta} \times L_{\gamma}$ satisfying:

$$
\left([a]_{\alpha}-x\right)+\left([b]_{\beta}-y\right)+\left([c]_{\gamma}-z\right)=0 \operatorname{Mod} 2,
$$

we can construct a quadruplet $\left(a^{\prime}, b^{\prime}, c_{1}^{\prime}, c_{2}^{\prime}\right)$ of generating curves of $\Gamma$ such that:

$$
\left[\Omega\left(a^{\prime}, b^{\prime}, c_{1}^{\prime}, c_{2}^{\prime}\right)\right]_{\alpha}=x, \quad\left[\Omega\left(a^{\prime}, b^{\prime}, c_{1}^{\prime}, c_{2}^{\prime}\right)\right]_{\beta}=y, \quad\left[\Omega\left(a^{\prime}, b^{\prime}, c_{1}^{\prime}, c_{2}^{\prime}\right)\right]_{\gamma}=z .
$$

By Proposition 4.5.1, the triple $(x, y, z)$ satisfies this condition precisely when: 


$$
\operatorname{Lift}_{\gamma}(z)^{-1} \operatorname{Lift}_{\alpha}(x) \operatorname{Lift}_{\beta}(y) \neq \operatorname{Id} .
$$

The result now follows.

\section{Constructing the local homeomorphism}

\subsection{Introduction}

Throughout this section, $(M, Q)$ will be a pointed, compact, three dimensional manifold of strictly negative sectional curvature, $(\tilde{M}, \tilde{Q})$ its universal cover, and $\Gamma=\langle\alpha, \beta\rangle$ a Schottky subgroup of $\pi_{1}(M, Q)$.

In this section, we will prove the main results of this paper. First we have:

Theorem 1.0.4 Suppose that $(M, Q)$ is a pointed, compact manifold of strictly negative sectional curvature. Let $(\Sigma, P)$ be a pointed, compact surface of hyperbolic type (i.e. of genus at least two $)$. Let $\theta: \pi_{1}(\Sigma, P) \rightarrow \pi_{1}(M, Q)$ be a homomorphism. Suppose that $\theta$ is nonelementary and may be lifted to a homomorphism $\hat{\theta}$ of $\pi_{1}(\Sigma, P)$ into the group $\widetilde{\mathrm{Homeo}}_{0}\left(\partial_{\infty} \tilde{M}\right)$. Then there exists an equivariant Plateau problem for $\theta$.

We then prove:

Theorem 1.0.5 If $\theta$ is non-elementary and lifts, then there exists a convex immersion $i$ : $\Sigma \rightarrow M$ such that:

$$
\theta=i_{*} .
$$

In the second part of this section, we define the notion of a $\pi_{1}(M, Q)$ structure, and prove an existence result for such structures over bound, marked pants. In the third part of this section, we state results which summarise the glueing technique used by Gallo, Kapovich and Marden in Sect. 8 of [4]. In the fourth section we provide a proof of Theorem 1.0.4. Finally, in the fifth section we show how to obtain convex solutions to the Plateau problem in Hadamard manifolds, and this permits us to prove Theorem 1.0.5.

\subsection{Constructing the solution over pants}

Let $(\Sigma, \theta, \beta)$ be a bound marked surface. Let $\tilde{\Sigma}$ be the universal cover of $\Sigma$. We define a $\pi_{1}(M, Q)$ structure over $(\Sigma, \theta, \beta)$ to be a local homeomorphism $\varphi: \tilde{\Sigma} \rightarrow \partial_{\infty} \tilde{M}$ such that:

(i) $\varphi$ is equivariant under the action of $\theta$, and

(ii) for every boundary component $C$ of $\tilde{\Sigma}$, the restriction of $\varphi$ to $C$ is a homeomorphism onto its image. In other words $\varphi(C)$ is a non self-intersecting curve.

Let $P_{0}$ be the base point of $\Sigma$, and let $\tilde{P}_{0}$ be the corresponding base point in the universal cover $\tilde{\Sigma}$ of $\Sigma$. Let $(C, P)$ be a pointed boundary component of $\Sigma$. Let $\eta$ be an element of $\pi_{1}\left(\Sigma, P_{0}, P\right)$, and let $\tilde{\eta}$ be the lift of $\eta$ such that:

$$
\tilde{\eta}(0)=\tilde{P}_{0}
$$

Let $\hat{P}_{\eta}$ be the endpoint of $\tilde{\eta} . \hat{P}_{\eta}$ is thus a lift of $P$. Viewing $C$ as a parameterized simple closed curve in $\Sigma$ leaving $P$, we define $\hat{C}_{\eta}$ to be the lift of $C$ starting from $\hat{P}_{\eta}$. The curve $C_{\eta}$ is thus a segment of one of the boundary components of $\tilde{\Sigma}$. Moreover, by definition:

$$
\hat{C}_{\eta}(0) \cdot\left(\eta^{-1} C \eta\right)=\hat{C}_{\eta}(1) .
$$


Since $\varphi \circ \hat{C}_{\eta}$ is non self-intersecting, it must avoid the fixed points of $\theta\left(\eta^{-1} C \eta\right)$ in $\partial_{\infty} \tilde{M}$. Consequently $\varphi \circ \hat{C}_{\eta}$ projects down to a closed curve in $\mathbb{T}_{\theta\left(\eta^{-1} C \eta\right)}$. We will denote this element by $[C]_{\eta, \varphi}$.

Let $\gamma$ and $\xi$ be two elements of $\pi_{1}\left(M, Q_{0}\right)$. Let $\xi$ be another element. The mapping $\xi$ sends $\gamma_{ \pm}$onto the fixed points $\delta_{ \pm}$of $\delta=\xi^{-1} \gamma \xi$. It follows that $\xi$ defines a homeomorphism from $\mathbb{T}_{\gamma}$ to $\mathbb{T}_{\delta}$, which we will also denote by $\xi$. Moreover for any $\alpha \in \pi_{1}(\Sigma)$ :

$$
\begin{aligned}
& \hat{C}_{\eta} \cdot \alpha=\hat{C}_{\eta \alpha} \\
& \Rightarrow \varphi\left(\hat{C}_{\eta} \cdot \alpha\right)=\varphi\left(\hat{C}_{\eta \alpha}\right) \\
& \Rightarrow\left(\varphi \circ \hat{C}_{\eta}\right) \cdot \theta(\alpha)=\left(\varphi \circ \hat{C}_{\eta \alpha}\right) \\
& \Rightarrow[C]_{\eta, \varphi} \cdot \theta(\alpha)=[C]_{\eta \alpha, \varphi} .
\end{aligned}
$$

We may thus define the element $\beta_{\varphi}(C)$ lying in $\mathbb{T}_{\beta(C)}$ such that, for all $\eta \in \pi_{1}\left(\Sigma, P_{0}, P\right)$ :

$$
\beta_{\varphi}(C)=[C]_{\eta, \varphi} \cdot \beta(\eta)^{-1} .
$$

We are now in a position to construct $\pi_{1}\left(M, Q_{0}\right)$ structures over bound, marked pants:

Proposition 5.2.1 Let $\left(M, Q_{0}\right)$ be a pointed three dimensional Hadamard manifold of strictly negative sectional curvature. Let $(T, \theta, \beta)$ be a bound marked pant with holonomy in $\pi_{1}(M$, $\left.Q_{0}\right)$. Suppose that the image of $\theta$ is a Schottky group.

Let $P_{0}$ be the base point of $T$ and let $\left(C_{j}, Q_{j}\right)_{1 \leqslant j \leqslant 3}$ be the three oriented boundary components of $T$. Let $\left(\xi_{j}\right)_{1 \leqslant j \leqslant 3}$ be a sash of $T$ with respect to the points $\left(Q_{j}\right)_{1 \leqslant j \leqslant 3}$ such that:

$$
\xi_{3}^{-1} C_{3}^{-1} \xi_{3}=\left(\xi_{1}^{-1} C_{1}^{-1} \xi_{1}\right)\left(\xi_{2}^{-1} C_{2}^{-1} \xi_{2}\right) .
$$

Let $\left(x_{1}, x_{2}, x_{3}\right) \in L_{\beta\left(C_{1}\right)} \times L_{\beta\left(C_{2}\right)} \times L_{\beta\left(C_{3}\right)}$ be a triplet such that:

$\operatorname{Lift}_{\theta\left(\xi_{3}^{-1} C_{3} \xi_{3}\right)}\left(x_{3} \cdot \beta\left(\xi_{3}\right)\right)^{-1} \operatorname{Lift}_{\theta\left(\xi_{1}^{-1} C_{1} \xi_{1}\right)}\left(x_{1} \cdot \beta\left(\xi_{1}\right)\right) \operatorname{Lift}_{\theta\left(\xi_{2}^{-1} C_{2} \xi_{2}\right)}\left(x_{2} \cdot \beta\left(\xi_{2}\right)\right) \neq \mathrm{Id}$.

Then, there exists a $\pi_{1}\left(M, Q_{0}\right)$ structure, $\varphi$, over $(T, \theta, \beta)$ such that, for each $i$ :

$$
\beta_{\varphi}\left(C_{i}\right)=x_{i}
$$

Proof Let $P_{T}$ be the base point of $T$. Let us denote $a=\xi_{1}^{-1} C_{1} \xi_{1}$ and $b=\xi_{2}^{-1} C_{2} \xi_{2}$. Let $\Gamma=\operatorname{Im}(\theta)$. Denote $\alpha=\theta(a)$ and $\beta=\theta(b)$. By Lemma 4.1.1, there exists an invariant domain $\Omega$ of $\Gamma$ adapted to the generators $(\alpha, \beta)$ such that:

$$
[\Omega]_{\alpha}=x_{1} \cdot \beta\left(\xi_{1}\right), \quad[\Omega]_{\beta}=x_{2} \cdot \beta\left(\xi_{2}\right), \quad[\Omega]_{\gamma}=x_{3} \cdot \beta\left(\xi_{3}\right) .
$$

Let $P_{0}$ be a point in the interior of $\hat{\Omega}$. Let $\pi: \hat{\Omega} \rightarrow \hat{\Omega} / \Gamma$ be the canonical projection. Let $\phi: \pi_{1}\left(\hat{\Omega} / \Gamma, \pi\left(P_{0}\right)\right) \rightarrow \Gamma$ be the unique isomorphism defined such that, for all $\gamma \in \Gamma$ and for any curve $c$ joining $P_{0}$ to $\gamma\left(P_{0}\right)$ :

$$
\phi([\pi \circ c])=\gamma .
$$

In particular, since $\Omega$ is adapted to the generators $(\alpha, \beta)$, we see that there exist boundary components $a^{\prime}$ and $b^{\prime}$ of $\hat{\Omega} / \Gamma$ and a sash $\left(c_{a^{\prime}}, c_{b^{\prime}}\right)$ of $\left(\hat{\Omega} / \Gamma, \pi\left(P_{0}\right)\right)$ with respect to the base points of $a^{\prime}$ and $b^{\prime}$ respectively such that:

$$
\phi\left(c_{a^{\prime}}^{-1} a^{\prime} c_{a^{\prime}}\right)=\alpha, \quad \phi\left(c_{b^{\prime}}^{-1} b^{\prime} c_{b^{\prime}}\right)=\beta .
$$

There exists a homeomorphism, $\varphi:\left(T, P_{T}\right) \rightarrow\left(\hat{\Omega} / \Gamma, \pi\left(P_{0}\right)\right)$ such that:

$$
\varphi_{*} a \sim c_{a^{\prime}}^{-1} a^{\prime} c_{a^{\prime}}, \quad \varphi_{*} b \sim c_{b^{\prime}}^{-1} b^{\prime} c_{b^{\prime}} .
$$


Consequently:

$$
\theta=\phi \circ \varphi_{*} .
$$

Let $\left(\tilde{T}, \tilde{P}_{T}\right)$ denote the pointed universal cover of $\left(T, P_{T}\right)$. The mapping $\varphi$ lifts to a unique pointed homeomorphism, $\tilde{\varphi}$, from $\left(\tilde{T}, \tilde{P}_{T}\right)$ to $\left(\hat{\Omega}, P_{0}\right)$ which is equivariant under the action of $\theta$. The curve $\tilde{\varphi} \circ C_{1}$ lies entirely in $\hat{\Omega}$. Thus, by definition of $[\Omega]_{\alpha}$ :

$$
\begin{aligned}
& {\left[C_{1}\right]_{\xi_{1}, \tilde{\varphi}}=[\Omega]_{\alpha}=x_{1} \cdot \beta\left(\xi_{1}\right)} \\
& \Rightarrow \beta_{\tilde{\varphi}}\left(C_{1}\right)=\left[C_{1}\right]_{\xi_{1}, \tilde{\varphi}} \cdot \beta\left(\xi_{1}\right)^{-1}=x_{1} .
\end{aligned}
$$

Similarly, $\beta_{\tilde{\varphi}}\left(C_{2}\right)=x_{2}$ and $\beta_{\tilde{\varphi}}\left(C_{3}\right)=x_{3}$. The mapping $\tilde{\varphi}$ is consequently the desired $\pi_{1}\left(M, Q_{0}\right)$ structure, and the result now follows.

\subsection{Joining the pants}

Let $\left(\Sigma_{i}, \theta_{i}, \beta_{i}\right)_{i \in\{1,2\}}$ be two bound marked surfaces with holonomy in $\pi_{1}(M, Q)$. For each $i$, let $\left(C_{i}, Q_{i}\right)$ be a pointed boundary component of $\Sigma_{i}$. Let $\psi:\left(C_{1}, Q_{1}\right) \rightarrow\left(C_{2}^{-1}, Q_{2}\right)$ be a homeomorphism and suppose that $\left(\Sigma_{1}, \theta_{1}, \beta_{1}\right)$ may be joined to $\left(\Sigma_{2}, \theta_{2}, \beta_{2}\right)$ along $\psi$. We recall that this means that $\beta_{1}\left(C_{1}\right)^{-1}=\beta_{2}\left(C_{2}\right)$ and consequently that:

$$
\mathbb{T}_{\beta_{1}\left(C_{1}\right)^{-1}}=\mathbb{T}_{\beta_{2}\left(C_{2}\right)} .
$$

We observe that:

$$
L_{\gamma^{-1}}=-L_{\gamma} .
$$

For each $i$, let $\varphi_{i}$ be a $\pi_{1}(M, Q)$ structure over $\left(\Sigma_{i}, \theta_{i}, \beta_{i}\right)$. We say that $\varphi_{1}$ may be joined to $\varphi_{2}$ along $\psi$ if and only if:

$$
-\beta_{1, \varphi_{1}}\left(C_{1}\right)=\beta_{2, \varphi_{2}}\left(C_{2}\right) .
$$

The glueing procedure described by Gallo, Kapovich and Marden in section 8 of [4] yields the following result:

Proposition 5.3.1 Let $(M, Q)$ be a compact, pointed, three dimensional manifold of strictly negative sectional curvature.

Let $\left(\Sigma_{i}, \theta_{i}, \beta_{i}\right)_{i \in\{1,2\}}$ be two bound marked surfaces with holonomy in $\pi_{1}(M, Q)$. For each $i$, let $\left(C_{i}, Q_{i}\right)$ be pointed boundary components of $\Sigma_{i}$ and let $\psi:\left(C_{1}, Q_{1}\right) \rightarrow\left(C_{2}^{-1}, Q_{2}\right)$ be a homeomorphism such that $\left(\Sigma_{1}, \theta_{1}, \beta_{1}\right)$ may be joined to $\left(\Sigma_{2}, \theta_{2}, \beta_{2}\right)$ along $\psi$.

Suppose that there exists, for each $i$, a $\pi_{1}(M, Q)$ structure $\varphi_{i}$ over $\left(\Sigma_{i}, \theta_{i}, \beta_{i}\right)$. If moreover, $\varphi_{1}$ may be joined to $\varphi_{2}$ along $\psi$, then there exists a $\pi_{1}(M, Q)$ structure $\varphi$ over $\left(\Sigma_{1}, \theta_{1}, \beta_{1}\right) \cup$ $\left(\Sigma_{2}, \theta_{2}, \beta_{2}\right)$ such that, after identifying objects in each of $\Sigma_{1}$ and $\Sigma_{2}$ with the corresponding objects in $\Sigma_{1} \cup \Sigma_{2}$, for each $i$ and for every pointed boundary component $\left(C^{\prime}, Q^{\prime}\right)$ of $\Sigma_{i}$ :

$$
\left(\beta_{1} \cup \beta_{2}\right)_{\varphi}\left(C^{\prime}\right)=\beta_{i, \varphi_{i}}\left(C^{\prime}\right) .
$$

Let $(\Sigma, \theta, \beta)$ be a bound marked surface with holonomy in $\pi_{1}(M, Q)$. Let $\left(C_{i}, Q_{i}\right)_{i \in\{1,2\}}$ be pointed boundary components of $\Sigma$. Let $\psi:\left(C_{1}, Q_{1}\right) \rightarrow\left(C_{2}^{-1}, Q_{2}\right)$ be an orientation reversing homeomorphism and suppose that $(\Sigma, \theta, \beta)$ may be joined to itself along $\psi$. Let $\varphi$ be a $\pi_{1}(M, Q)$ structure over $(\Sigma, \theta, \beta)$. We say that $\varphi$ may be joined to itself along $\psi$ if and only if:

$$
-\beta_{\varphi}\left(C_{1}\right)=\beta_{\varphi}\left(C_{2}\right)
$$


Once again, the glueing procedure described by Gallo, Kapovich and Marden in section 8 of [4] permits us to obtain the following analogue of Lemma 5.3.1:

Proposition 5.3.2 Let $(M, Q)$ be a compact, pointed, three dimensional manifold of strictly negative sectional curvature.

Let $(\Sigma, \theta, \beta)$ be a bound, marked surface with holonomy in $\pi_{1}(M, Q)$. Let $\left(C_{i}, Q_{i}\right)_{i \in\{1,2\}}$ be pointed boundary components of $\Sigma$. Let $\psi:\left(C_{1}, Q_{1}\right) \rightarrow\left(C_{2}^{-1}, Q_{2}\right)$ be a homeomorphism such that $(\Sigma, \theta, \beta)$ may be joined to itself along $\psi$.

Suppose that there exists, for each $i$, a $\pi_{1}(M, Q)$ structure, $\varphi$, over $(\Sigma, \theta, \beta)$. If, moreover, $\varphi$ may be joined to itself along $\psi$, then there exists a $\pi_{1}(M, Q)$ structure $\varphi^{\prime}$ over $\bigcirc(\Sigma, \theta, \beta)$ such that, after identifying objects in $\Sigma$ with the corresponding objects in $\bigcirc \Sigma$, for every pointed boundary component $\left(C^{\prime}, Q^{\prime}\right)$ of $\Sigma$ :

$$
(\bigcirc \beta)_{\varphi^{\prime}}\left(C^{\prime}\right)=(\bigcirc \beta)_{\varphi}(C) .
$$

\subsection{The construction of a local homeomorphism}

We are now in a position to prove Theorem 1.0.4:

Proof of Theorem 1.0.4 By Lemma 3.1.1, there exist $2 g-2$ bound, marked pants $\left(T_{i}, \theta_{i}\right.$, $\left.\beta_{i}\right)_{1 \leqslant i \leqslant 2 g-2}$ with holonomy in $\pi_{1}(M, Q)$ such that the image of every $\theta_{i}$ is a Schottky group, and:

$$
(\Sigma, \theta) \cong \bigodot_{i=1}^{g}\left(\underset{i=1}{2 g-2}\left(T_{i}, \theta_{i}, \beta_{i}\right)\right) .
$$

Let $\pi: \widetilde{\operatorname{Homeo}}_{0}\left(\partial_{\infty} \tilde{M}\right) \rightarrow \operatorname{Homeo}_{0}\left(\partial_{\infty} \tilde{M}\right)$ be the canonical projection. For every $i$, using the lifting, $\hat{\theta}$, of $\theta$, we may construct liftings $\hat{\theta}_{i}$ and $\hat{\beta}_{i}$ of $\theta_{i}$ and $\beta_{i}$ such that:

(i) for all $i, \pi \circ \hat{\theta}_{i}=\theta_{i}$ and $\pi \circ \hat{\beta}_{i}=\beta_{i}$, and

(ii) the $\left(T_{i}, \hat{\theta}_{i}, \hat{\beta}_{i}\right)_{1 \leqslant i \leqslant 2 g-2}$ may be joined along the same edges as the $\left(T_{i}, \theta_{i}, \beta_{1}\right)_{1 \leqslant i \leqslant 2 g-2}$. For each $i$, we label the boundary components of $T_{i}$ with distinct numbers from 1 to 3 . For all $1 \leqslant i \leqslant 2 g-2,1 \leqslant j \leqslant 3$, we denote by $\left(C_{i, j}, Q_{i, j}\right)$ the $j$ th pointed boundary component of $T_{i}$. Let $\alpha$ be the involution (having no fixed points) of $A=\{1, \ldots, 2 g-2\} \times\{1,2,3\}$ such that, for all $(i, j) \in A$, the boundary component $\left(C_{i, j}, Q_{i, j}\right)$ is joined to $\left(C_{\alpha(i, j)}, Q_{\alpha(i, j)}\right)$ in the above decomposition. For all $i, j$, we define $E_{i, j} \in L_{\beta\left(C_{i, j}\right)}$ such that:

(i) for all $(i, j), \operatorname{Lift}_{\beta_{i}\left(C_{i, j}\right)} E_{i, j} \neq \hat{\beta}_{i}\left(C_{i, j}\right)$, and

(ii) for all $(i, j), E_{i, j}=-E_{\alpha(i, j)}$.

Choose $1 \leqslant i \leqslant 2 g-2$. Let $\mathcal{P}_{i}$ be the base point of $T_{i}$. Let $\left(\xi_{j}\right)_{1 \leqslant j \leqslant 3}$ be a binding sash of $T_{i}$ with respect to the points $\left(Q_{i, j}\right)_{1 \leqslant j \leqslant 3}$ such that:

$$
\xi_{3}^{-1} C_{i, 3} \xi_{3}=\left(\xi_{1}^{-1} C_{i, 1} \xi_{1}\right)\left(\xi_{2}^{-1} C_{i, 2} \xi_{2}\right) \text {. }
$$

For each $j$, let us denote $\gamma_{j}=\xi_{j}^{-1} C_{i, j} \xi_{j}$ and $a_{j}=E_{i, j} \cdot \beta\left(\xi_{j}\right)$. Let $\Delta \in \widetilde{\operatorname{Homeo}}_{0}\left(\partial_{\infty} \tilde{M}\right)$ be the element of $\pi^{-1}$ (Id) that is different to the identity. We recall that $\Delta$ commutes with every element of $\widetilde{H o m e o}\left(\partial_{\infty} \tilde{M}\right)$. For each $j$, we have:

$$
\begin{aligned}
\operatorname{Lift}_{\theta\left(\gamma_{j}\right)}\left(a_{j}\right) & =\beta\left(\xi_{j}\right)^{-1}\left[\operatorname{Lift}_{\beta\left(\xi_{j}\right) \theta\left(\gamma_{j}\right) \beta\left(\xi_{j}\right)^{-1}}\left(E_{i, j}\right)\right] \beta\left(\xi_{j}\right) \\
& =\hat{\beta}\left(\xi_{j}\right)^{-1} \operatorname{Lift}_{\beta\left(C_{i, j}\right)}\left(E_{i, j}\right) \hat{\beta}\left(\xi_{j}\right) \\
& =\hat{\beta}\left(\xi_{j}\right)^{-1} \hat{\beta}\left(C_{i, j}\right) \Delta \hat{\beta}\left(\xi_{j}\right) \\
& =\hat{\theta}\left(\gamma_{j}\right) \Delta .
\end{aligned}
$$


Thus:

$$
\begin{aligned}
\operatorname{Lift}_{\theta\left(\gamma_{3}\right)}\left(a_{3}\right)^{-1} \operatorname{Lift}_{\theta\left(\gamma_{1}\right)}\left(a_{1}\right) \operatorname{Lift}_{\theta\left(\gamma_{2}\right)}\left(a_{2}\right) & =\hat{\theta}\left(\gamma_{3}\right)^{-1} \hat{\theta}\left(\gamma_{1}\right) \hat{\theta}\left(\gamma_{2}\right) \Delta^{3} \\
& =\hat{\theta}\left(\gamma_{3}^{-1} \gamma_{1} \gamma_{2}\right) \Delta^{3} \\
& =\Delta^{3} \\
& =\Delta .
\end{aligned}
$$

Thus, by Proposition 5.2.1, there exists a $\pi_{1}(M, Q)$ structure $\varphi_{i}$ over $\left(T_{i}, \theta_{i}, \beta_{i}\right)$ such that, for each $j$ :

$$
\beta_{i, \varphi_{i}}\left(C_{i, j}\right)=E_{i, j} .
$$

By definition of $\left(E_{i, j}\right)_{(i, j) \in A}$, these $\pi_{1}(M, Q)$ structures may all be joined to each other, and the existence of a $\pi_{1}(M, Q)$ structure over $(\Sigma, \theta)$ now follows using a process of induction and Propositions 5.3.1 and 5.3.2. The result now follows.

\subsection{Convex subsets of Hadamard manifolds}

We now require the following results concerning convex sets:

Proposition 5.5.1 Let $p$ be a point in $\partial_{\infty} \tilde{M}$ and let $\Omega$ be a neighbourhood of $p$ in $\partial_{\infty} \tilde{M}$. There exists a complete convex subset $X$ of $\tilde{M}$ such that:

$$
p \notin \partial_{\infty} X, \quad \Omega^{c} \subseteq \partial_{\infty} X .
$$

Proof Let $K \geqslant k>0$ be such that the sectional curvature of $\tilde{M}$ is contained in $[-K,-k]$. For all $q$ in $\tilde{M}$, for $r \in \partial_{\infty} \tilde{M}$ and for $\theta \in(0, \pi)$, we define $\Omega_{\theta}(r ; q)$ as in section 2.3. By Theorem 3.1 of [1], there exists $\pi / 2>\psi>0$ such that, for all $q \in \tilde{M}$ and for all $r \in \partial_{\infty} \tilde{M}$, there exists a complete convex subset $X$ of $\tilde{M}$ such that:

$$
\partial_{\infty} \Omega_{\psi}(r ; q) \subseteq \partial_{\infty} X
$$

Let $q_{0}$ be any point in $\tilde{M}$. Let $\gamma$ be the unique geodesic running from $q_{0}$ to $p$, normalised such that $\gamma(0)=q_{0}$ and $\gamma(\infty)=p$. Let $\delta \in \mathbb{R}^{+}$be such that:

$$
\partial_{\infty} \Omega_{\delta}\left(p ; q_{0}\right) \subseteq \Omega \text {. }
$$

Let us define $B \in \mathbb{R}^{+}$such that:

$$
\Delta_{k}(B, \pi-\delta)<\psi .
$$

Let $q_{1}=\gamma(B)$. Let $X$ be a complete convex subset of $\tilde{M}$ such that:

$$
\partial_{\infty} \Omega_{\psi}\left(q_{1} ; \gamma(-\infty)\right) \subseteq \partial_{\infty} X
$$

Thus, by Lemma 2.3.1:

$$
\Omega^{c} \subseteq \partial_{\infty} \Omega_{\pi-\delta}(p ; \gamma(-\infty)) \subseteq \partial_{\infty} \Omega_{\psi}\left(q_{1} ; \gamma(-\infty)\right) \subseteq \partial_{\infty} X
$$

The result now follows.

This result now permits us to show:

Lemma 5.5.2 Let $D$ be a closed subset of $\partial_{\infty} \tilde{M}$. There exists a complete strictly convex subset $X$ with $C^{1}$ boundary in $\tilde{M}$ such that:

$$
D=\partial_{\infty} X
$$


Proof For all $p \in D^{c}$, by Proposition 5.5.1, there exists a complete convex subset $X_{p}$ of $\partial_{\infty} \tilde{M}$ such that $p \notin \partial_{\infty} X_{p}$ and $D \subseteq \partial_{\infty} X_{p}$. We define $\hat{X}$ by:

$$
\hat{X}=\bigcap_{p \in C^{c}} X_{p} .
$$

$\hat{X}$ is a complete convex subset of $\tilde{M}$ and $\partial_{\infty} \hat{X}=D$. In particular, $\hat{X}$ is non-empty. For $\epsilon \in \mathbb{R}^{+}$, let $\Sigma_{\epsilon}$ be the surface obtained by moving $\partial \hat{X}$ a distance $\epsilon$ normally along geodesics. $\Sigma_{\epsilon}$ is strictly convex, is of type $C^{1}$ and bounds a complete convex subset $X$ of $\tilde{M} . X$ is thus the desired subset, and the result now follows.

We are now in a position to prove Theorem 1.0.5:

Proof of Theorem 1.0.5 We give an elementary account of a technique described by Labourie in [5]. Since $\Sigma$ is compact, and since $\varphi$ is $\theta$-equivariant, there exists a finite open cover $\mathcal{A}=\left(\Omega_{i}\right)_{1 \leqslant i \leqslant k}$ of $\Sigma$ such that, if $\tilde{\mathcal{A}}=\left(\Omega_{i, \gamma}\right)_{1 \leqslant i \leqslant k, \gamma \in \pi_{1}(\Sigma, P)}$ is the lifting of $\mathcal{A}$ to $\tilde{\Sigma}$, then, for every $\Omega \in \tilde{\mathcal{A}}$ :

(i) the closure of $\Omega$ in $\tilde{\Sigma}$ is homeomorphic to a closed disc, and

(ii) for every $\Omega \in \tilde{\mathcal{A}}$, the restriction of $\varphi$ to $\bar{\Omega}$ is a homeomorphism onto its image.

By Lemma 5.5.2, for every $i$, we may find a family $\left(X_{i, \gamma}\right)_{\gamma \in \pi_{1}(\Sigma, P)}$ of complete strictly convex subsets with $C^{1}$ boundary in $\tilde{M}$ such that:

(i) $\left(X_{i, \gamma}\right)_{\gamma \in \pi_{1}(\Sigma, P)}$ is $\theta$-equivariant. In other words, for all $\gamma, \delta \in \pi_{1}(\Sigma, P)$ :

$$
X_{i, \gamma \delta}=X_{i, \gamma} \cdot \theta(\delta)
$$

and

(ii) for all $\gamma \in \pi_{1}(\Sigma, P)$ :

$$
\partial_{\infty} X_{1, \gamma}=\varphi\left(\Omega_{i, \gamma}\right)^{c} .
$$

We may assume moreover that the boundaries of these sets intersect transversally in the region that will be of interest to us. Using these convex sets, we construct a family $\left(P_{i, \gamma}\right)_{1 \leqslant i \leqslant k, \gamma \in \pi_{1}(\Sigma, P)}$ of polygonal subsets of $\tilde{\Sigma}$ and a family $\left(\psi_{i, \gamma}\right)_{1 \leqslant i \leqslant k, \gamma \in \pi_{1}(\Sigma, P)}$ of immersions such that:

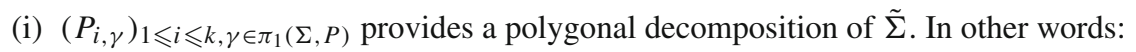

$$
\tilde{\Sigma}=\underset{\substack{1 \leqslant i \leqslant k \\ \gamma \in \pi_{(\Sigma, P)}}}{\cup} P_{i, \gamma},
$$

and two polygons in this family only intersect if they share a common boundary component;

(ii) the family $\left(P_{i, \gamma}\right)_{1 \leqslant i \leqslant k, \gamma \in \pi_{1}(\Sigma, P)}$ is equivariant. In other words, for each $i$, and for all $\gamma, \delta \in \pi_{1}(\Sigma, P)$ :

$$
P_{i, \gamma \delta}=P_{i, \gamma} \cdot \delta
$$

(iii) for each $i$ and for all $\gamma, \psi_{i, \gamma}$ is a homeomorphism from $P_{i, \gamma}$ onto a subset of the boundary of $X_{i, \gamma}$ in $\tilde{M}$, and

(iv) the family $\left(\psi_{i, \gamma}\right)_{1 \leqslant i \leqslant k, \gamma \in \pi_{1}(\Sigma, P)}$ is $\theta$-equivariant. In other words, for each $i$, and for all $\gamma, \delta \in \pi_{1}(\Sigma, P)$, and for all $p \in P_{i, \gamma}$ :

$$
\psi_{i, \gamma \delta}(p \cdot \delta)=\psi_{i, \gamma}(p) \cdot \theta(\delta) .
$$


Joining together the elements of $\left(\psi_{i, \gamma}\right)_{1 \leqslant i \leqslant k, \gamma \in \pi_{1}(\Sigma, P)}$, we obtain a $\theta$-equivariant locally strictly convex immersion $\hat{\psi}$ of $\tilde{\Sigma}$ into $\tilde{M}$ such that if $\vec{n}$ is the Gauss-Minkowski mapping sending $U \tilde{M}$ into $\partial_{\infty} \tilde{M}$, then:

$$
\vec{n} \circ \hat{\psi}=\varphi .
$$

Taking quotients, we obtain a locally strictly convex immersion $\psi$ of $\Sigma$ into $M$ which realises $\theta$. Finally, since $\Sigma$ is compact, by deforming $\psi$ slightly, we may suppose that it is also smooth and the result now follows.

\section{A Appendix A: Homeomorphism equivalence of Schottky groups}

\section{A.1 Introduction}

Throughout this appendix, $(M, Q)$ will be a pointed three dimensional Hadamard manifold and $\Gamma=\langle\alpha, \beta\rangle$ a Schottky subgroup of $\operatorname{Isom}(M, Q)$.

In this appendix, we provide a proof of the equivalence up to homeomorphisms of the Schottky groups that we will be using.

We define $\operatorname{Fix}(\Gamma)$, the fixed point set of $\Gamma$ by:

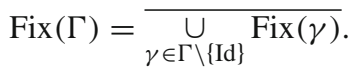

We define a reduced word over $(\alpha, \beta)$ to be a sequence $\gamma=\left(\gamma_{k}\right)_{1 \leqslant k \leqslant n}$ of elements of $\left\{\alpha^{ \pm 1}, \beta^{ \pm 1}\right\}$ which does not contain any of the subwords $\alpha \alpha^{-1}, \alpha^{-1} \alpha, \beta \beta^{-1}$ or $\beta^{-1} \beta$. In other words, a reduced word is the shortest length word expressing the corresponding element of $\Gamma$. Let $W_{\alpha, \beta}$ denote the set of all reduced words over $(\alpha, \beta)$ of finite length. Let $W_{\alpha, \beta}^{n}$ denote the subset of $W_{\alpha, \beta}$ consisting of words of length $n$. Let $W_{\alpha, \beta}^{\infty}$ denote the set of all reduced words over $(\alpha, \beta)$ of infinite length. For all $m \geqslant n$, we define the truncation map $T_{n}: W_{\alpha, \beta}^{m} \rightarrow W_{\alpha, \beta}^{n}$ to be the map which sends a word of length $m$ to the word given by its first $n$ letters. This definition is trivially also valid when $m=\infty$. We give $W_{\alpha, \beta}$ the discrete topology, and we then give $W_{\alpha, \beta}^{\infty}$ the coarsest topology with respect to which every $T_{n}$ is continuous. In other words, a basis of open sets of $W_{\alpha, \beta}^{\infty}$ is given by:

$$
\mathcal{B}=\cup_{n \in \mathbb{N}}\left\{T_{n}^{-1}(\{\boldsymbol{\gamma}\}) \text { s.t. } \boldsymbol{\gamma} \text { is of length } n\right\} .
$$

This topology is trivially Hausdorff. Moreover, since the alphabet is finite, it is not difficult to show that $W_{\alpha, \beta}^{\infty}$ is compact with respect to this topology.

The first result of this appendix is the construction of a canonical homeomorphism from $W_{\alpha, \beta}^{\infty}$ to Fix $(\Gamma)$. In order to explicitly describe the homeomorphism, we are required to construct a few more objects. Let $\left(C_{\alpha}^{ \pm}, C_{\beta}^{ \pm}\right)$be generating circles for $\Gamma$ in $\partial_{\infty} \tilde{M}$ with respect to the generators $(\alpha, \beta)$. We define the set $S_{1}$ by $S_{1}=\left\{C_{\alpha}^{ \pm}, C_{\beta}^{ \pm}\right\}$and we define $S_{n}$ for $n \geqslant 2$ inductively by:

$$
S_{n}=\left(\underset{\gamma \in\left\{\alpha^{ \pm 1}, \beta^{ \pm 1}\right\}}{\cup} \gamma S_{n-1}\right) \backslash \bigcup_{k=0}^{n-1} S_{n-1} .
$$


For all $n \geqslant 2$, and for all $C \in S_{n}$, there exists a unique $C^{\prime} \in S_{1}$ such that $C \subseteq \operatorname{Int}\left(C^{\prime}\right)$. We thus orient $C$ such that $\operatorname{Int}(C) \subseteq \operatorname{Int}\left(C^{\prime}\right)$. We observe that, for all $n$, and for any distinct $C, C^{\prime} \in S_{n}$, the interiors of $C$ and $C^{\prime}$ are also distinct.

We define the mapping $D_{1}: W_{\alpha, \beta}^{1} \rightarrow S_{1}$ by:

$$
D_{1}\left(\alpha^{ \pm 1}\right)=C_{\alpha}^{ \pm}, \quad D_{1}\left(\beta^{ \pm 1}\right)=C_{\beta}^{ \pm} .
$$

We define $D_{n}$ for $n \geqslant 2$ such that, for all $\gamma=\left(\gamma_{k}\right)_{1 \leqslant k \leqslant n}$ in $W_{\alpha, \beta}^{n}$ :

$$
D_{n}(\boldsymbol{\gamma})=D_{1}\left(\gamma_{n}\right) \cdot \gamma_{n-1} \cdot \ldots \cdot \gamma_{1}
$$

We then define $D$ over $W_{\alpha, \beta}$ such that it restricts to $D_{n}$ over each $W_{\alpha, \beta}^{n}$. We observe that, for all $n, D_{n}$ defines a bijection between $W_{\alpha, \beta}^{n}$ and $S_{n}$. We now obtain the following result:

Lemma A.1.1 Suppose that the sectional curvature of $M$ is bounded above by $-k<0$. For all $\boldsymbol{\gamma}=\left(\gamma_{n}\right)_{n \in \mathbb{N}}$ in $W_{\alpha, \beta}^{\infty}$, there exists a unique point $\mathcal{P}(\gamma) \in \operatorname{Fix}(\Gamma)$ such that the sequence $\left(\operatorname{Int}\left(D\left(T_{n}(\boldsymbol{\gamma})\right)\right)\right)_{n \in \mathbb{N}}$ converges towards $\{\mathcal{P}(\boldsymbol{\gamma})\}$ in the Hausdorff topology. Moreover, $\mathcal{P}(\boldsymbol{\gamma})$ is contained in $\operatorname{Int}\left(D\left(T_{n}(\gamma)\right)\right)$ for every $n$, and $\mathcal{P}$ defines a homeomorphism between $W_{\alpha, \beta}^{\infty}$ and $\operatorname{Fix}(\Gamma)$.

Extending this to a homeomorphism between $\partial_{\infty} M$ and an abstract space over which $\Gamma$ acts in a trivial manner, we obtain the following result which tells us that all the Schottky groups that we will be studying are essentially equivalent to Schottky subgroups of $\mathbb{P} S L(2, \mathbb{C})$ :

Lemma A.1.2 Suppose that the sectional curvature of $M$ is bounded above by $-k<0$. Let $\Gamma \subseteq \operatorname{Isom}_{0}(M)$ and $\Gamma^{\prime} \subseteq \operatorname{Isom}_{0}\left(M^{\prime}\right)$ be Schottky subgroups. There exists an isomorphism $\phi: \Gamma \rightarrow \Gamma^{\prime}$ and a homeomorphism $\Phi: \partial_{\infty} M \rightarrow \partial_{\infty} M^{\prime}$ such that, for all $\gamma \in \Gamma:$

$$
\Phi \circ \gamma=\varphi(\gamma) \circ \Phi
$$

In the second part of this appendix, we prove Lemma A.1.1, and then in the third part we show how this result may be used to prove Lemma A.1.2.

\section{A.2 The fixed point set of a Schottky group}

In this subsection, we prove Lemma A.1.1. We begin with the following more elementary result concerning Hadamard manifolds of strictly negative sectional curvature:

Proposition A.2.1 Let $M$ be a Hadamard manifold of sectional curvature bounded above by $-k<0$. Let $U M$ be the unitary bundle over $M$ and let $\pi: U M \rightarrow M$ be the canonical projection. Let $\left(v_{n}\right)_{n \in \mathbb{N}}$ be a sequence of vectors in $U M$ such that $\left(\pi \circ v_{n}\right)_{n \in \mathbb{N}}$ converges to a point $p_{0}$ in $\partial_{\infty} M$. For all $n$, let $\gamma_{n}$ be the geodesic in $M$ leaving $\pi \circ v_{n}$ with velocity $v_{n}$. After extraction of a subsequence, at least one of $\left(\gamma_{n}(-\infty)\right)_{n \in \mathbb{N}}$ and $\left(\gamma_{n}(+\infty)\right)_{n \in \mathbb{N}}$ converges also to $p_{0}$.

Proof Let $q_{0}$ be a point in $M$. For all $n$, let us denote $p_{n}=\pi \circ v_{n}, D_{n}=d\left(q_{0}, p_{n}\right)$, and let $q_{n} \in \partial_{\infty} M$ be the unique point such that $p_{n}$ lies on the geodesic segment joining $q_{0}$ to $q_{n}$. Trivially $\left(q_{n}\right)_{n \in \mathbb{N}}$ converges to $p_{0}$ and $\left(D_{n}\right)_{n \in \mathbb{N}}$ tends to infinity. By Lemma 2.3.1, without loss of generality, for all $n$ :

$$
\gamma_{n}(+\infty) \in \partial_{\infty} \Omega_{\Delta_{k}}\left(D_{n}, \pi / 2\right)\left(q_{n} ; q_{0}\right) .
$$

Since $\left(\Delta_{k}\left(D_{n}, \pi / 2\right)\right)_{n \in \mathbb{N}}$ tends to zero, the result follows. 
We now define the subset $X$ of $M$ by:

$$
X=M \backslash\left(\operatorname{Int}\left(D_{\alpha}^{+}\right) \cup \operatorname{Int}\left(D_{\alpha}^{-}\right) \cup \operatorname{Int}\left(D_{\beta}^{+}\right) \cup \operatorname{Int}\left(D_{\beta}^{-}\right)\right) .
$$

Trivially:

$$
\partial_{\infty} X=\partial_{\infty} M \backslash\left(\operatorname{Int}\left(C_{\alpha}^{+}\right) \cup \operatorname{Int}\left(C_{\alpha}^{-}\right) \cup \operatorname{Int}\left(C_{\beta}^{+}\right) \cup \operatorname{Int}\left(C_{\beta}^{-}\right)\right) .
$$

For all $\gamma \in \Gamma \backslash\{\mathrm{Id}\}$, let $\gamma^{-}$and $\gamma^{+}$be the repulsive and attractive fixed points of $\gamma$ respectively, and let $g_{\gamma}$ be the unique geodesic joining $\gamma^{-}$to $\gamma^{+}$. We define $G_{\Gamma}$ by:

$$
G_{\Gamma}=\underset{\gamma \in \Gamma}{\cup} g_{\gamma}(\mathbb{R}) .
$$

We obtain the following result:

Proposition A.2.2 The intersection $X \cap G_{\Gamma}$ is bounded in $M$.

Proof Suppose the contrary. There exists a sequence $\left(p_{n}\right)_{n \in \mathbb{N}} \in X \cap G_{\Gamma}$ and $p_{0} \in \partial_{\infty} M$ such that $\left(p_{n}\right)_{n \in \mathbb{N}}$ converges to $p_{0}$. For all $n$, let $\gamma_{n} \in \Gamma$ be such that $p_{n}$ lies in $g_{\gamma_{n}}$. For all $n$, let $\gamma_{n, \pm}$ be the fixed points of $\gamma_{n}$. By Lemma A.2.1, we may assume that $\left(\gamma_{n,+}\right)_{n \in \mathbb{N}}$ tends to $p_{0}$, in which case $p_{0} \in \operatorname{Fix}(\Gamma)$. However, $p_{0} \in \partial_{\infty} X$, which is absurd, since $\operatorname{Fix}(\Gamma)$ and $\partial_{\infty} X$ are disjoint. The result now follows.

We define the evaluation map Eval : $W_{\alpha, \beta} \rightarrow \Gamma$ such that, for all $\boldsymbol{\gamma}=\left(\gamma_{k}\right)_{1 \leqslant k \leqslant n}$ :

$$
\operatorname{Eval}(\boldsymbol{\gamma})=\gamma_{n} \cdot \ldots \cdot \gamma_{1}
$$

We have the following elementary result:

Lemma A.2.3 For every $\boldsymbol{\gamma}=\left(\gamma_{k}\right)_{1 \leqslant k \leqslant n}$ in $W_{\alpha, \beta}$, we have:

$$
\operatorname{Ext}\left(D_{1}\left(\gamma_{n}^{-1}\right)\right) \cdot \operatorname{Eval}(\gamma) \subseteq \operatorname{Int}\left(D_{1}\left(\gamma_{1}\right)\right) .
$$

Proof We prove this result by induction on the length of $\gamma$. The result follows immediately from the definition of generating circles when $\gamma$ is of length 1 . We suppose now that the result is true when $\boldsymbol{\gamma}$ is of length $n$. Let $\boldsymbol{\gamma}=\left(\gamma_{k}\right)_{1 \leqslant k \leqslant n}$ be a reduced word of length $n+1$ over $(\alpha, \beta)$. By the induction hypothesis, we have:

$$
\operatorname{Ext}\left(D_{1}\left(\gamma_{n+1}^{-1}\right)\right) \cdot \operatorname{Eval}(\gamma) \subseteq \operatorname{Int}\left(D_{1}\left(\gamma_{2}\right)\right) \cdot \gamma_{1} .
$$

Since $\gamma$ is a reduced word, $\gamma_{2} \neq \gamma_{1}^{-1}$. Consequently:

$$
\begin{aligned}
& \operatorname{Int}\left(D_{1}\left(\gamma_{2}\right)\right) \subseteq \operatorname{Ext}\left(D_{1}\left(\gamma_{1}^{-1}\right)\right) \\
& \Rightarrow \operatorname{Int}\left(D_{1}\left(\gamma_{2}\right)\right) \cdot \gamma_{1} \subseteq \operatorname{Int}\left(D_{1}\left(\gamma_{1}\right)\right) .
\end{aligned}
$$

The result now follows.

This yields the following two corollaries concerning $D(\gamma)$ for $\gamma$ in $W_{\alpha, \beta}$. First, we have:

Corollary A.2.4 For every $\boldsymbol{\gamma}=\left(\gamma_{k}\right)_{1 \leqslant k \leqslant n}$ in $W_{\alpha, \beta}$ :

$$
\operatorname{Int}(D(\gamma))=\operatorname{Ext}\left(D_{1}\left(\gamma_{n}^{-1}\right)\right) \cdot \operatorname{Eval}(\gamma) .
$$

Proof This follows from Lemma A.2.3 and the fact that $D(\gamma)$ is oriented such that its interior is contained in the interior of the unique circle in $S_{1}$ in which it lies. 
Next we have:

Corollary A.2.5 For every $\gamma=\left(\gamma_{k}\right)_{k \in \mathbb{N}}$ in $W_{\alpha, \beta}^{\infty}$ and for all $n \in \mathbb{N}$ :

$$
\operatorname{Int}\left(D\left(T_{n+1}(\gamma)\right)\right) \subseteq \operatorname{Int}\left(D\left(T_{n}(\gamma)\right)\right) .
$$

Proof Indeed, by Corollary A.2.4:

$$
\operatorname{Int}\left(D\left(T_{n+1}(\gamma)\right)\right)=\operatorname{Int}\left(D_{1}\left(\gamma_{n+1}\right)\right) \cdot \operatorname{Eval}\left(T_{n}(\gamma)\right) .
$$

Since $\gamma$ is a reduced word, $\gamma_{n+1} \neq \gamma_{n}^{-1}$, and so:

$$
\operatorname{Int}\left(D_{1}\left(\gamma_{n+1}\right)\right) \subseteq \operatorname{Ext}\left(D_{1}\left(\gamma_{n}\right)\right) .
$$

The result follows by applying $\operatorname{Eval}\left(T_{n}(\gamma)\right)$ to both sides and using Corollary A.2.5.

We define $W_{\alpha, \beta}^{0}$ to be the set of all reduced words $\gamma=\left(\gamma_{k}\right)_{1 \leqslant k \leqslant n}$ in $W_{\alpha, \beta}$ such that $\gamma_{n} \neq \gamma_{1}^{-1}$. We have the following result:

Proposition A.2.6 $\|\operatorname{Eval}(\gamma)\|$ tends to infinity as the length of $\gamma$ tends to infinity in $W_{\alpha, \beta}^{0}$.

Proof Suppose the contrary. There exists $K>0$ and infinitely many distinct elements $\left(\gamma_{n}\right)_{n \in \mathbb{N}}=\left(\operatorname{Eval}\left(\gamma_{n}\right)\right)_{n \in \mathbb{N}}$ in $\Gamma$ such that, for all $n,\left\|\gamma_{n}\right\| \leqslant K$. Since, for all $n, \gamma_{n} \in W_{\alpha, \beta}^{0}$, by Lemma A.2.3, we may suppose that there exist two distinct circles $C_{1}$ and $C_{2}$ in $S_{1}$ such that, for all $n$ :

$$
\operatorname{Ext}\left(C_{1}\right) \cdot \gamma_{n} \subseteq \operatorname{Int}\left(C_{2}\right)
$$

Consequently, if $\eta_{n}$ is the geodesic fixed by $\gamma_{n}$, then $\eta_{n}$ intersects $X$ non-trivially. Thus, for all $n$, there exists $p_{n} \in X \cap G_{\Gamma}$ such that:

$$
d\left(p_{n}, p_{n} \cdot \gamma_{n}\right) \leqslant K
$$

Let $p$ be an arbitrary point in $M$. By compactness, there exists $B>0$ such that, for all $q \in X \cap G_{\Gamma}$ :

$$
d(p, q) \leqslant B
$$

Thus, for all $n$ :

$$
\left.d\left(p, p \cdot \gamma_{n}\right)\right) \leqslant 2 B+K .
$$

Consequently, by compactness, the set $\{p\} \cdot \Gamma$ has a concentration point in $\tilde{M}$, which is absurd. The result now follows.

For $\gamma$ an element of $W_{\alpha, \beta}^{\infty}$, we define the sequence $\left(l_{n}(\gamma)\right)_{n \in \mathbb{N}}$ such that, for all $n$ :

$$
l_{n}(\gamma)=\operatorname{Sup}\left\{k \text { s.t. } 1 \leqslant k \leqslant n \& \gamma_{k} \neq \gamma_{1}^{-1}\right\} .
$$

We now obtain the following partial proof of Lemma A.1.1:

Proposition A.2.7 Suppose that the sectional curvature of $M$ is bounded above by $-k<0$. For all $\boldsymbol{\gamma}=\left(\gamma_{n}\right)_{n \in \mathbb{N}}$ in $W_{\alpha, \beta}^{\infty}$ such that $\left(l_{n}(\gamma)\right)_{n \in \mathbb{N}}$ tends to infinity, there exists a unique point $\mathcal{P}(\gamma) \in \operatorname{Fix}(\Gamma)$ such that the sequence $\left(\operatorname{Int}\left(D\left(T_{n}(\gamma)\right)\right)\right)_{n \in \mathbb{N}}$ converges towards $\{\mathcal{P}(\gamma)\}$ in the Hausdorff topology. 
Proof For all $n$, we define $\boldsymbol{\mu}_{n} \in W_{\alpha, \beta}^{l_{n}(\gamma)}$ and $\boldsymbol{v}_{n} \in W_{\alpha, \beta}^{n-l_{n}(\gamma)}$ such that:

$$
\boldsymbol{\mu}_{n}=T_{l_{n}(\gamma)}(\boldsymbol{\gamma}), \quad T_{n}(\boldsymbol{\gamma})=\boldsymbol{v}_{n} \boldsymbol{\mu}_{n} .
$$

For all $n$, and for all $1 \leqslant k \leqslant l_{n}(\boldsymbol{\gamma})$, we denote by $\mu_{n, k}$ the $k$ th letter of $\boldsymbol{\mu}_{n}$. By corollary A.2.5, for all $n$ :

$$
\operatorname{Int}\left(D\left(T_{n}(\boldsymbol{\gamma})\right)\right), \quad \operatorname{Int}\left(D\left(\boldsymbol{\mu}_{n+1}\right)\right) \subseteq \operatorname{Int}\left(D\left(\boldsymbol{\mu}_{n}\right)\right) .
$$

We define $\Omega$ and $\Delta$ as in section 2.3. By compactness, there exists $\epsilon>0$ such that, for all $p^{\prime} \in X \cap G_{\Gamma}$, for all $q \in \operatorname{Fix}(\Gamma)$ and for all $C \in S_{1}$ :

$$
C \cap \Omega_{\epsilon}\left(q ; p^{\prime}\right)=\emptyset .
$$

Let $\theta>0$ be an angle. By compactness, there exists $\varphi>0$ such that, for all $p, p^{\prime} \in$ $X \cap G_{\Gamma}$ :

$$
\partial_{\infty} \Omega_{\varphi}(q ; p) \subseteq \partial_{\infty} \Omega_{\theta}\left(q ; p^{\prime}\right)
$$

By Proposition A.2.6, there exists $N \in \mathbb{N}$ such that, for $n \geqslant N$ :

$$
\Delta_{k}\left(\left\|\operatorname{Eval}\left(\boldsymbol{\mu}_{n}\right)\right\|, \pi-\epsilon\right)<\varphi .
$$

Let us denote by $\eta_{n}$ the unique geodesic preserved by $\operatorname{Eval}\left(\boldsymbol{\mu}_{n}\right)$. It follows that $\eta_{n}$ intersects $X$ non-trivially. Let $p_{n}$ be any point in $X \cap \eta_{n}$. Let $p_{n}^{-}$and $p_{n}^{+}$be the repulsive and attractive fixed points respectively of $\operatorname{Eval}\left(\boldsymbol{\mu}_{n}\right)$. We have:

$$
\operatorname{Ext}\left(D_{1}\left(\mu_{n, l_{n}(\gamma)}^{-1}\right)\right) \subseteq \partial_{\infty} \Omega_{\pi-\epsilon}\left(p_{n}^{+} ; p_{n}\right) .
$$

Thus, by Lemma 2.3.1 and Lemma A.2.3:

$$
\begin{aligned}
\operatorname{Int}\left(D\left(\boldsymbol{\mu}_{n}\right)\right)= & \operatorname{Ext}\left(D_{1}\left(\mu_{n, l_{n}(\gamma)}^{-1}\right)\right) \cdot \operatorname{Eval}(\boldsymbol{\mu}) \\
& \subseteq \partial_{\infty} \Omega_{\varphi}\left(p_{n}^{+} ; p_{n}\right) \\
& \subseteq \partial_{\infty} \Omega_{\theta}\left(p_{n}^{+} ; p\right) .
\end{aligned}
$$

Thus, since $\theta$ is arbitrary, the diameter of $\left(\operatorname{Int}\left(D\left(\boldsymbol{\mu}_{n}\right)\right)\right)_{n \in \mathbb{N}}$ with respect to any given metric on $\partial_{\infty} M$ tends to zero.

Since $\left(\overline{\operatorname{Int}\left(D\left(\boldsymbol{\mu}_{n}\right)\right)}\right)_{n \in \mathbb{N}}$ is a nested sequence of compact sets, its intersection is nonempty. Since its diameter is zero, the intersection contains at most 1 point. Since, for all $n$, $\operatorname{Int}\left(D\left(T_{n}\left(\boldsymbol{\gamma}_{n}\right)\right)\right) \subseteq \operatorname{Int}\left(D\left(\boldsymbol{\mu}_{n}\right)\right)$, and the result follows.

We may then use this result to obtain:

Proposition A.2.8 Suppose that the sectional curvature of $M$ is bounded above by $-k<$ 0 . For all $\gamma$ in $W_{\alpha, \beta}^{\infty}$, there exists a unique point $\mathcal{P}(\gamma) \in \operatorname{Fix}(\Gamma)$ such that the sequence $\left(\operatorname{Int}\left(D\left(T_{n}(\boldsymbol{\gamma})\right)\right)\right)_{n \in \mathbb{N}}$ converges towards $\{\mathcal{P}(\boldsymbol{\gamma})\}$ in the Hausdorff topology.

Proof By Proposition A.2.7, it suffices to prove the result when $\left(l_{n}(\gamma)\right)_{n \in \mathbb{N}}$ is bounded. Let $k$ be such that, for all $n$ :

$$
l_{n}(\boldsymbol{\gamma}) \leqslant k
$$

By definition of $\left(l_{n}(\gamma)\right)_{n \in \mathbb{N}}$, for all $m \geqslant k$ :

$$
\gamma_{m}=\gamma_{1}^{-1}
$$


We thus define $\boldsymbol{\mu}=T_{k}(\boldsymbol{\gamma})$, and we define $\boldsymbol{\gamma}^{\prime} \in W_{\alpha, \beta}^{\infty}$ such that:

$$
\gamma=\gamma^{\prime} \mu .
$$

Trivially, for all $n, l_{n}\left(\boldsymbol{\gamma}^{\prime}\right)=n$. By Corollary A.2.4:

$$
\operatorname{Int}\left(D\left(T_{n}\left(\boldsymbol{\gamma}^{\prime}\right)\right)\right) \cdot \operatorname{Eval}(\boldsymbol{\mu})=\operatorname{Int}\left(D\left(T_{n+k}(\boldsymbol{\gamma})\right)\right) .
$$

We define $\mathcal{P}(\boldsymbol{\gamma})=\mathcal{P}\left(\boldsymbol{\gamma}^{\prime}\right) \cdot \operatorname{Eval}(\boldsymbol{\mu})$, and the result now follows by Proposition A.2.7.

We now obtain a proof of Lemma A.1.1:

Proof of Lemma A.1.1 Existence and uniqueness of $\mathcal{P}$ follow from Propositions A.2.7 and A.2.8. By corollary A.2.5, for all $\gamma$ and for all $n$ :

$$
\mathcal{P}(\boldsymbol{\gamma}) \in \operatorname{Int}\left(D\left(T_{n}(\boldsymbol{\gamma})\right)\right) .
$$

Continuity of $\mathcal{P}$ now follows by a diagonal argument.

Let $p$ be a point in $\operatorname{Fix}(\Gamma)$. For all $n, p$ lies in the union of the interiors of the circles in $S_{n}$. Since $D_{n}$ defines a bijection between $W_{\alpha, \beta}^{n}$ and $S_{n}$, there exists $\gamma_{n} \in W_{\alpha, \beta}^{n}$ such that:

$$
p \in \operatorname{Int}\left(D\left(\gamma_{n}\right)\right) \text {. }
$$

By compactness, there exists $\gamma \in W_{\alpha, \beta}^{\infty}$ such that $\left(\boldsymbol{\gamma}_{n}\right)_{n \in \mathbb{N}}$ tends to $\gamma$. For $m \in \mathbb{N}$ and for all $n$ sufficiently large, $T_{m}(\boldsymbol{\gamma})=T_{m}\left(\boldsymbol{\gamma}_{n}\right)$. Thus, by Corollary A.2.5:

$$
p \in \operatorname{Int}\left(D\left(\gamma_{n}\right)\right) \subseteq \operatorname{Int}\left(T_{m}(\gamma)\right) .
$$

By taking limits, it follows that $\mathcal{P}(\boldsymbol{\gamma})=p$, and surjectivity follows.

Let $\boldsymbol{\gamma}$ and $\boldsymbol{\gamma}^{\prime}$ be two points in $W_{\alpha, \beta}^{\infty}$ such that $\mathcal{P}(\boldsymbol{\gamma})=\mathcal{P}(\boldsymbol{\gamma})=p$. For all $n$, the interiors of $D\left(T_{n}(\gamma)\right)$ and $D\left(T_{n}\left(\gamma^{\prime}\right)\right)$ intersect non-trivially and thus coincide. Thus, for all $n$, $T_{n}(\gamma)=T_{n}\left(\gamma^{\prime}\right)$. Injectivity now follows.

Since $\mathcal{P}$ is a bijective continuous mapping between two compact sets it is a homeomorphism, and the result now follows.

\section{A.3 Homeomorphism equivalence of Schottky groups}

Let $\left(C_{\alpha}^{ \pm}, C_{\beta}^{ \pm}\right)$be generating circles of $\Gamma$ with respect to the generators $(\alpha, \beta)$. We define $\Omega \subseteq \partial_{\infty} M$ by:

$$
\Omega=\partial_{\infty} M \backslash\left(\operatorname{Int}\left(C_{\alpha}^{+}\right) \cup \operatorname{Int}\left(C_{\alpha}^{-}\right) \cup \operatorname{Int}\left(C_{\beta}^{+}\right) \cup \operatorname{Int}\left(C_{\beta}^{-}\right)\right) .
$$

We define the continuous mapping $\Phi: \Omega \times \Gamma \rightarrow \partial_{\infty} M$ by:

$$
\Phi(x, \gamma)=x \cdot \gamma \text {. }
$$

We define the equivalence relation $\sim$ over $\Omega \times \Gamma$ such that:

$$
(x, \gamma) \sim(y, \eta) \Leftrightarrow \Phi(x, \gamma)=\Phi(y, \eta) .
$$

Since $\Omega$ is a fundamental domain for the action of $\Gamma$, we find that $(x, \gamma) \sim(y, \eta)$ if and only if either $x=y$ and $\gamma=\eta$ or:

(i) $x, y \in \partial \Omega$, and

(ii) there exists $\mu \in\left\{\alpha^{ \pm 1}, \beta^{ \pm 1}\right\}$ such that: 


$$
(x, \gamma)=\left(y \cdot \mu, \mu^{-1} \cdot \eta\right)
$$

$\Omega \times \Gamma / \sim$ is a Hausdorff space and that $\Phi$ quotients down onto a homeomorphism of $\Omega \times \Gamma / \sim$ onto its image. Using Lemma A.1.1, it is fairly trivial to show that $\partial_{\infty} M=\operatorname{Im}(\Phi) \cup \operatorname{Fix}(\Gamma)$. We now define the space $\Sigma_{\Gamma}$ by:

$$
\Sigma_{\Gamma}=(\Omega \times \Gamma / \sim) \cup W_{\alpha, \beta}^{\infty}
$$

For all $n$, and for all $\gamma \in W_{\alpha, \beta}^{n}$, we define the subset $U_{\gamma}$ of $\Sigma_{\Gamma}$ by:

$$
U_{\boldsymbol{\gamma}}=\operatorname{Int}\left(\underset{\substack{\boldsymbol{\eta} \in W_{\alpha, \beta} \text { s.t } \\ l(\boldsymbol{\eta}) \geqslant n, T_{n}(\boldsymbol{\eta})=\boldsymbol{\gamma}}}{\cup} \Omega \times\{\operatorname{Eval}(\boldsymbol{\eta})\} / \sim\right) \cup\left\{\boldsymbol{\gamma}^{\prime} \in W_{\alpha, \beta}^{\infty} \text { s.t. } T_{n}\left(\boldsymbol{\gamma}^{\prime}\right)=\boldsymbol{\gamma}\right\} .
$$

If $\tau$ denotes the topology of $\Omega \times \Gamma / \sim$, then $\tau \cup\left\{U_{\gamma}\right.$ s.t. $\left.\gamma \in W_{\alpha, \beta}\right\}$ defines a base for a topology over $\Sigma_{\Gamma}$. This topology is Hausdorff and restricts to the initial topologies over $\Omega \times \Gamma / \sim$ and $W_{\alpha, \beta}^{\infty}$. It is fairly trivial to show that $\Sigma_{\Gamma}$ is compact. Moreover, if $\gamma \in W_{\alpha, \beta}^{\infty}$ and if $\left(p_{n}, \gamma_{n}\right)_{n \in \mathbb{N}}$ is a sequence in $\Omega \times \Gamma / \sim$ converging to $\gamma$, then, for all $k \in \mathbb{N}$, there exists $N \in \mathbb{N}$ such that:

$$
n \geqslant N \Rightarrow T_{k}\left(\gamma_{n}\right)=T_{k}(\gamma)
$$

We now define the mapping $\Psi: \Sigma_{\Gamma} \rightarrow \partial_{\infty} \tilde{M}$ by:

$$
\Psi(p)=\left\{\begin{array}{l}
\mathcal{P}(p) \text { if } p \in W_{\alpha, \beta}^{\infty}, \\
\Phi(p) \text { if } p \in \Omega \times \Gamma / \sim .
\end{array}\right.
$$

Using Lemma A.1.1 we may show that the mapping $\Psi$ is continuous and bijective. Since $\Sigma_{\Gamma}$ is compact, $\Phi$ is a homeomorphism. We have thus constructed a topological space homeomorphic to the sphere. Moreover, there exists a canonical action of $\Gamma$ over this space with which $\Psi$ intertwines. We now prove Lemma A.1.2:

Proof of Lemma A.1.2 Let $(\alpha, \beta)$ and $\left(\alpha^{\prime}, \beta^{\prime}\right)$ be generators of $\Gamma$ and $\Gamma^{\prime}$ respectively. Let $\left(C_{\alpha}^{ \pm}, C_{\beta}^{ \pm}\right)$and $\left(C_{\alpha}^{\prime \pm}, C_{\beta}^{\prime \pm}\right)$ be generating circles of $\Gamma$ and $\Gamma^{\prime}$ respectively with respect to these generators. We define $\Omega \subseteq \partial_{\infty} M$ and $\Omega^{\prime} \subseteq \partial_{\infty} M^{\prime}$ as before. We define $\phi: \Gamma \rightarrow \Gamma^{\prime}$ such that $\phi(\alpha)=\alpha^{\prime}$ and $\phi(\beta)=\beta^{\prime}$. Let $\hat{\Phi}: \Omega \rightarrow \Omega^{\prime}$ be a homeomorphism such that, for all $p \in C_{\alpha}^{-}$and for all $q \in C_{\beta}^{-}$:

$$
\begin{aligned}
& \hat{\Phi}(p) \cdot \alpha^{\prime}=\hat{\Phi}(p \cdot \alpha), \\
& \hat{\Phi}(q) \cdot \beta^{\prime}=\hat{\Phi}(q \cdot \alpha) .
\end{aligned}
$$

There exists a unique extension of $\hat{\Phi}$ to a mapping from $\Sigma_{\Gamma}$ to $\Sigma_{\Gamma^{\prime}}$ which intertwines with $\phi$. We define $\Psi_{1}: \Sigma_{\Gamma} \rightarrow \partial_{\infty} M$ and $\Psi_{2}: \Sigma_{\Gamma} \rightarrow \partial_{\infty} M^{\prime}$ as before. For all $\gamma \in \Gamma$, the following diagram trivially commutes: 


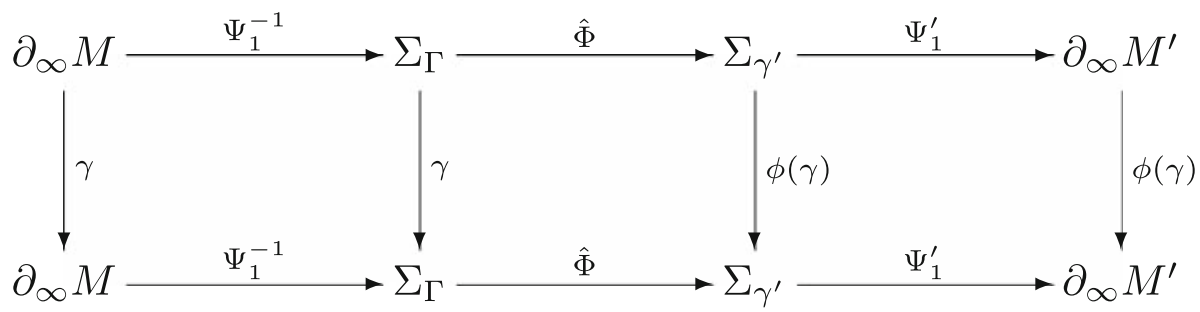

The mapping $\Phi=\Psi_{1}^{\prime} \circ \hat{\Phi} \circ \Psi_{1}^{-1}$ is thus the desired homeomorphism, and the result now follows.

Open Access This article is distributed under the terms of the Creative Commons Attribution Noncommercial License which permits any noncommercial use, distribution, and reproduction in any medium, provided the original author(s) and source are credited.

\section{References}

1. Anderson, M.: The Dirichlet Problem at infinity for manifolds of negative curvature. J. Differ. Geom 18, 701-702 (1983)

2. Ballman, W., Gromov, M., Schroeder, V.: Manifolds of nonpositive curvature, Progress in Mathematics, 61, Birkhäuser, Boston (1985)

3. Friberg, B.: A topological proof of a theorem of Kneser. Proc. Am. Math. Soc. 39, 421-425 (1973)

4. Gallo, D., Kapovich, M., Marden, A.: The monodromy groups of Schwarzian equations on closed Riemann surfaces. Ann. Math. 151(2), 625-704 (2000)

5. Labourie, F.: Un lemme de Morse pour les surfaces convexes. Invent. Math. 141, 239-297 (2000)

6. Le Roux, F.: Thèse doctorale, Grenoble (1997)

7. Smith, G.: Thèse de doctorat, Paris (2004)

8. Smith, G.: Hyperbolic Plateau Problems, Preprint, Orsay (2005)

9. Smith, G.: A Homomorphism for Braid Groups in the Sphere (in preparation) 